\title{
1 Phages weaponize their bacteria with biosynthetic gene clusters
}

2 Anna Dragoš1\#, Aaron J.C. Andersen¹, Carlos N. Lozano-Andrade1, Paul J. Kempen², Ákos T.

3 Kovács ${ }^{1}$ and Mikael Lenz Strube ${ }^{1 \#}$

4

$5{ }^{1}$ Department of Biotechnology and Biomedicine, Technical University of Denmark, Søltofts Plads bldg.

$6 \quad$ 221, DK-2800 Kgs Lyngby, Denmark

$7 \quad{ }^{2}$ Department of Health Technology, Technical University of Denmark, Produktionstorvet bldg. 423, DK-

82800 Kgs Lyngby, Denmark

9

10 \#Corresponding authors:

11 Mikael Lenz Strube (milst@dtu.dk)

12 Anna Dragoš (adragos@dtu.dk)

13

14 ORCID numbers:

15 AD: 0000-0003-4136-986X

16 AJCA: 0000-0002-3876-7480

17 CNLA: 0000-0003-2805-4505

18 PJK: 0000-0003-2179-2257

19 ATK: 0000-0002-4465-1636

20 MLS: 0000-0003-0905-5705

21

22 Keywords:

23 Biosynthetic gene cluster, genome mining, phages, bacteriocins, interbacterial competition 


\section{ABSTRACT}

25 Bacteria produce many different specialized metabolites, which are encoded by biosynthetic gene clusters

26 (BGCs). Despite high industrial relevance owing to broad bioactive potential of these metabolites, their

27 ecological roles remain largely unexplored. We analyze all available genomes for BGCs of phage origin.

28 The BGCs predominantly reside within temperate phages infecting certain commensal and pathogenic

29 bacteria. Nearly all phage BGCs encode bacteriocins, which appear to serve as a strong proxy for phage

30 specificity. Using the gut-associated bacterium Bacillus subtilis, we demonstrate how a temperate phage

31 equips its host with a functional BGC, providing it with a competitive fitness advantage over close relatives.

32 Therefore, certain temperate phages use BGCs to weaponize their bacteria against close relatives, leading

33 to evolutionary benefits from lysogeny to the infected host, and hence, to the phage itself. Our study is a

34 large step towards understanding the natural role of specialized metabolites, as well as mutualistic phage-

35 host relationships. 


\section{Introduction}

Apart from molecules directly involved in basic metabolism, microbes produce a plethora of specialized

compounds, the so-called specialized metabolites. These metabolites mediate microbial ecological

41 interactions, but also supply medical ${ }^{1}$ and biotech industries ${ }^{2}$ with novel biological activities. Despite a

42 long tradition of industrial exploitation, the natural role of most specialized metabolites remains largely

43 unknown ${ }^{3}$, although there is evidence that some of them act as chemical weapons in competitive

44 interaction ${ }^{4,5}$.

Enzymatic pathways required to produce specialized metabolites are encoded by biosynthetic gene

clusters (BGCs). Although BGCs differ both structurally and functionally, major groups with similar

47 biosynthetic function are found, e.g. polyketide synthases (PKSs) and non-ribosomal peptide synthetases

48 (NRPSs), which are extremely large (10-100 kb), multi-enzyme complexes synthesizing products with such

properties as: antibiotic, signaling, immunosuppressive and biosurfactant activities ${ }^{6,7}$. Ribosomal peptide

natural products (RPNPs or RiPPs) are mostly represented by bacteriocins, recognized for their role in

51 interbacterial competition ${ }^{8}$. and their biosynthetic gene clusters are smaller compared to PKSs and NRPSs.

52 The presence (and expression) of a BGC on a bacterial genome can determine its pathogenicity ${ }^{9,10}$, the

53 potential for its success in an ecological niche ${ }^{11}$, and recently, we are becoming aware of the importance

54 of specialized metabolites in human microbiomes in the balance between health and disease ${ }^{12}$. Regardless

55 of the particular niche of a microorganism, it is evident that it will be locked in a perpetual state of

56 chemical warfare with others ${ }^{4}$ and given the antibiotic activity of many specialized metabolites, we

57 assume that specialized metabolites are crucial for the assembly of microbiomes. It has been proposed

58 that stability in microbiomes is promoted when interactions are few and predominantly competitive ${ }^{5}$,

59 suggesting that not only the individual bacteria, but the community as a whole benefits from hosting a

60 BGC that encode an antagonistic compound(s). Bacteriocins are particularly interesting in this context as 
61 they are uniformly bactericidal in contrast to more complicated specialized metabolites which may have

62 entirely divergent effects depending on the concentration ${ }^{13}$.

63 Progress in metagenomics along with excellent computational tools to predict BGCs ${ }^{14}$ have allowed us to

64 characterize the genetic potential for production of specialized metabolites in microbiomes, as well as

65 identify bacteria which are the main hosts of $\mathrm{BGCs}{ }^{15-17}$. For instance, in soil microbiomes, Acidobacteria

tend to be the richest in BGCs, mainly synthesizing polyketides, non-ribosomal peptides or terpenes,

67 however the Actinobacteria phylum contains multiple 'outliers' with extremely high (>20) BGC cargo ${ }^{16}$.

Moreover, the abundance of BGCs of soil microbiomes also varies depending on vegetation and soil

depth $^{16}$. In the healthy human gut microbiome, Bacteroides genus serves as the major BGC-carrier, with

saccharides being the most abundant specialized metabolites, while common gut-associated genera like

71 Escherichia, Lactobacillus, Haemophilus or Enterococcus, appear limited in BGC cargo ${ }^{15}$. In the human oral

72 microbiomes, the Firmicutes are the main carriers of BGCs, with oligosaccharide-encoding clusters and

73 RiPPs as the major BGC types ${ }^{12}$. Although current interdisciplinary approaches allow us to identify the key

74 BGC types and their producers, understanding the natural function of specialized metabolites and the eco-

75 evolutionary factors behind their distribution in microbial communities remains challenging.

76 In certain bacterial genera, BGCs are commonly associated with mobile genetic elements (MGEs) ${ }^{18}$, the

77 so-called flexible genome. For instance large BGCs are commonly found on plasmids of Pseudonocardia ${ }^{18}$,

78 but whether, or how, being part of MGEs translate into structure and ecological function of the specialized

79 metabolite remains unknown. This knowledge can be one of the large steps towards understanding the

80 role of specialized metabolites in bacterial communities and possibly also their role in balance between

81 health and dysbiosis ${ }^{19}$.

82 Amongst MGEs, phages are considered the most abundant biological agents on Earth. Phages have a 
of healthy gut microbiota ${ }^{21}$. Lytic phages hijack the DNA and protein synthesis machinery of the host,

85 rapidly multiply and eventually lyse the host cell to release progeny virions. Temperate phages can integrate their DNA into the host chromosome using specific or unspecific attachment sites (att) and replicate with the host bacterium as a prophage. Most bacterial genomes carry at least one prophage ${ }^{22}$, and prophage elements can occupy up to $20 \%$ of the entire bacterial genome ${ }^{23}$.

Phages can encode virulence factors ${ }^{24,25}$, toxins ${ }^{26}$ and other compounds potentially valuable for the host.

For instance, the SP $\beta$ prophage present in some strains of a soil, plant and gut bacterium Bacillus subtilis ${ }^{28}$,

91 encodes for an S-linked glycocin called sublancin ${ }^{29,30}$. The sublancin cluster $(5 \mathrm{~kb})$ contains a precursor peptide (sunA), posttranslational modification enzymes (sunS, bdbA, bdbB), a self-immunity protein (sunl), and a transporter (sunT) that ensures the export of the bacteriocin into extracellular space ${ }^{31}$. Sublancin presumably enters the cells using the PTS-glucose specific transport system ${ }^{32}$, and upon entry, negatively affects DNA replication, transcription and RNA translation ${ }^{33}$. Mutants deprived of SP $\beta$, the entire sublancin cluster or just the immunity gene sunl, are inhibited by isogenic strains that carry intact SP $\beta^{34}$. This

97 prophage can be found in closely related but geographically dispersed $B$. subtilis isolates, always occupying the same position in the chromosome ${ }^{28}$. The contribution of sublancin to ecological success of SP $\beta$ remains an open question. In the same line of thought, we do not know if other phages/prophages carry BGCs and whether the products of these BGCs may share structural or functional features. Such knowledge would contribute to our understanding of the natural role of bacterial BGCs, and would also facilitate mining and utilization of new specialized metabolites.

The aim this study was to explore all high-quality phage and prophage genomes for the presence of BGCs using a large-scale bioinformatics approach. We demonstrate that BGCs are more likely to be found within genomes of temperate phages which infect commensal/pathogenic bacterial hosts. The study also shows that nearly all phage-BGCs encode bacteriocins, suggesting they may serve as chemical weapons provided by phages to their bacterial hosts, hence increasing benefits from lysogeny. This hypothesis is 
bioRxiv preprint doi: https://doi.org/10.1101/2020.10.01.322628; this version posted October 1,2020. The copyright holder for this preprint (which was not certified by peer review) is the author/funder, who has granted bioRxiv a license to display the preprint in perpetuity. It is made available under aCC-BY-ND 4.0 International license. experimentally confirmed, revealing the competitive benefits from bacteriocin-carrying phage acquisition

109 by a natural $B$. subtilis isolate. Comparison of host phylogeny, with phage and BGC relatedness shows that 110 bacteriocins serve as a good indicator of phage host range. Our work contributes significantly to 111 understanding of ecological role of specialized metabolites, as well as cooperative relationship between 112 phages and their bacteria. 


\section{Results}

114 Biosynthetic gene clusters (BGCs) are extremely rare within virion-derived genomes

115 To assess the contribution of phages to the genetic pool of microbial specialized metabolites, we began

116 from a basic question: can biosynthetic gene clusters (BGCs) that encode for specialized metabolites be

117 found on phage genomes? To answer this question, all complete phage genomes available in the PATRIC

118 3.6.2 database (10063 in total) were subjected to BGC detection by antiSMASH 4 (see methods).

119 Interestingly, only approximately $0.07 \%$ of all phages (69 in total) carried BGCs (Fig. 1A). These phage-

120 encoded BGCs will be referred to as pBGCs (phage-encoded biosynthetic gene clusters).

121 The vast majority (64) of pBGCs were found in temperate phages (for which lifestyle was confirmed

122 experimentally in the literature) and only five pBGCs were carried by a lytic phage (life style confirmed

123 experimentally in the literature) (Suppl. dataset 1 ). We also noticed that phages infecting certain bacterial

124 genera were overrepresented among the pBGC hits, compared to relative distribution of phage hosts in

125 PATRIC database (Suppl. dataset 1, Suppl. Fig. 1A). These genera where Escherichia (represented solely by

126 Escherichia coli species), Mannheimia, Enterobacter and Shigella (Fig. 2A, Suppl. Fig. 1A).

127 It is important to note that number of BGC hits per phage genome was restricted to 1 or none in all cases.

128 This initial analysis suggested that pBGCs are extremely rare, and that they are more likely to reside within

129 host-associated (temperate) phages infecting particular bacterial species.

\section{BGCs are more abundant in prophage elements compared to virions}

132 Since the previous analysis suggested that pBGCs may be more common within temperate phages, the

133 pBGCs encoded by prophage elements were investigated. As the pBGC-positive phage pool is largely 134 overrepresented by Escherichia coli phages (Suppl. dataset 1), the aim was to analyze a more diverse set 
of (pro)phage genomes. Prophage regions were predicted and extracted from all high-quality bacterial genomes available in the NCBI database, and subjected these to BGC detection by antiSMASH 4 (see methods) (Fig. 1B). We found that the majority of analyzed bacterial genomes (67.5\% out of 15184 total genomes analyzed) carried at least one prophage element, and only approximately $3 \%$ of these prophage regions (307 in total) contained a BGC (Fig. 1B, Suppl. dataset 2) corresponding to approximately $2 \%$ of all bacterial genomes.

It was again observed that certain host species (including three out of four that were also 'popular' host genera of pBGC-carrying virions) were overrepresented among pBGC-carrying prophage elements compared to relative abundance of these genera in the entire genome database (Suppl. dataset 2, Suppl. Fig 1B). Specifically, nearly $90 \%$ of all pBGC-carrying prophages were associated with 11 genera of human/animal commensals and pathogens: Escherichia (in which all but 1 isolate belonged to E. coli), Mannheimia (where all isolates belonged to Mannhemia heamolytica), Bacillus, Pasteurella, Haemophilus, Lactobacillus, Enterococcus, Enterobacter, Listeria, Aeromonas and Klebsiella. All, apart from the latter two, seemed to be several-fold overrepresented within our pBGC dataset (Fig. 2A, Suppl. dataset 2, Suppl. Fig 1B). However, not all representatives of these genera had pBGCs. In addition, pBGC were clearly overrepresented in prophages of certain species, e.g. B. subtilis which is in the minority (20\%) of all Bacillus genomes in $\mathrm{NCBI}$, but it makes up for the majority (74 \%) of pBGC hits within Bacillus genus; Lactobacillus brevis ( $4 \%$ of all lactobacilli but majority (67\%) of pBGC hits within Lactobacillus genus), or Enterococcus faecalis ( $22 \%$ of all enterococci and over $90 \%$ of pBGC hits within corresponding genus) (Suppl. dataset 2, Suppl. Fig 1B).

In the vast majority of pBGC-carrying bacteria, the prophage carried only one BGCs, with the notable exceptions being some isolates of $M$. haemolytica (8 isolates), E. coli (7 isolates) and L. brevis (1 isolate) carrying 2 pBGCs. A single genome belonging to Alkaliphilus metalliredigens carried three pBGCs, all of which were identical (Supp. dataset 2). 
Since total number of phages were substantially higher in pBGC hosts (Fig. 2B, Mann-Whitney U-test, referred to as gBGC for core-genome-encoded biosynthetic gene cluster) were assessed (Suppl. dataset

(Fig 2B, Mann-Whitney U-test, $\mathrm{P}=0.0011$ ). numbers of gBGCs, phages appeared as a major or even a sole source of biosynthetic gene clusters (Fig. temperate phages, infecting certain species of human/animal commensals or pathogens, with limited gBGC cargo.

\section{The vast majority of prophage-encoded BGCs are bacteriocins}

172 We then investigated if prophage-encoded BGCs share structural/functional features. Indeed, according

173 to antiSMASH prediction, nearly all pBGC were assigned to bacteriocins ( $99.3 \%$ ), with some stratification

174 onto glycocins $(n=22,6.8 \%)$ and lantipeptides $(n=2,0.6 \%)$. To investigate how many of the antiSMASH-

175 predicted pBGC belonged to experimentally confirmed/studied bacteriocins, the pBGCs were compared

176 with the BACTIBASE database (see methods). The vast majority of pBGCs had poor matches in BACTIBASE,

177 most having protein similarities and alignment lengths well below $60 \%$ or lower (Fig. 3). Examples of

178 pBGCs of high similarity to experimentally confirmed bacteriocins were: sublancin (22 hits in B. subtilis),

179 enterocin (5 hits in L. monocytogenes) and carocin (Pectobactrerium carotovorum (GCF_000294535.1)).

180 Linocin was found in a close to full length, but highly dissimilar, version in Nocardia terpenica

181 (GCF_002568625.1) and E. coli (GCF_001901005.1 and GCF_900636075.1). 
182 Although the vast majority of pBGCs were bacteriocins, we have also found several exceptions. For instance, 2 pBGCs found in two separate Pseudomonas aeruginosa genomes contained a partial NRPS-like cluster with a single AMP-binding domain as well as a siderophore receptor and a sigma-70 polymerase sigma factor (Suppl. dataset 2). Two pBGCs found within 2 Mycobacterium temperate phages encoded for ectoine which may play a role in osmoadaptation, a pBGC found on the pseudo-lysogenic phage Streptomyces phage ZL12 was annotated as a Type 3 PKS, while a pBGC found in a lytic Roseobacter phage encoded for homo-serine lactone, which is a molecule involved in cell-to-cell communication (Suppl. dataset 1).

These results suggest that phages can be the source of different BGC types, however the vast majority of pBGCs are bacteriocins - putative weapons of competition against closely related bacteria.

\section{Phages weaponize their hosts with BGCs through lysogeny}

Since pBGCs are more commonly found in temperate phages, it was hypothesized they may play an

195 important role during lysogeny. As the vast majority of pBGCs are bacteriocins, they could serve as 196 biological weapons during competition of the host bacterium with its closely-related neighbors, thereby

197 increasing fitness benefits from lysogeny for both host and phage. This hypothesis was tested using a $B$. 198 subtilis model system, by transferring a pBGC carrying phage from one natural isolate to another and 199 monitoring fitness benefits from such pBGC-phage infection (Fig. 4A). The isolate MB8_B7 (CP045821) ${ }^{35}$ 200 served as a phage donor, as it is a natural host of an SP $\beta$ phage, which carries the biosynthetic gene cluster 201 of the antimicrobial glycocin, sublancin (Fig. 4A, Suppl. Fig. 2A). The pBGC-negative isolate P9_B1 was used 202 as a receiver strain lacking SP $\beta$ phage in the genome $(C P 045811)^{35}$.

203 The genome sequence of the MB8_B7 prophage was identical to the SP $\beta$-phage found in the lab strain $B$. 204 subtilis 168, except that the MB8_B7 does not contain ICEBs1, shown to interfere with the lytic cycle of 
205

206

207

208

209

210

211

212

213

214

215

216

217

218

219

220

221

222

223

224

225

226

227

$\mathrm{SP} \beta^{36}$ (see methods). It was confirmed that deletion of SP $\beta$ from MB8_B7 provoked sensitivity towards the wild-type (WT) MB8_B7 (Fig. 4A, Suppl. Fig. 2B). Importantly, sublancin could be detected in the spent medium of MB8_B7 but not in its $\triangle S P \beta$ derivative (Fig. 4B).

Next, SP $\beta$ virions were isolated from MB8_B7 culture (see methods), confirmed its identity by Nanopore sequencing (see methods) (Suppl. Fig. 6A) and used it to infect the naturally pBGC-free isolate P9_B1 (Fig. 4A, Suppl. Fig. 2CD). As anticipated, the P9_B1 ${ }^{S P \beta}$ became antagonistic towards its P9_B1 ancestor (Fig. Suppl. Fig. 2C). The P9_B1 ${ }^{\mathrm{SP} \beta}$ was also able to produce sublancin (Fig. 4AB). These results clearly demonstrated that the phage-encoded BGC and its biological activity can be easily transferred between two strains via phage infection (Fig. 4AB).

To further examine benefits from pBGC acquisition, the P9_B1 and P9_B1 ${ }^{\mathrm{SP} \beta}$ were labelled with fluorescent reporters (see methods; Suppl. Fig. 3A), and followed their growth in co-cultures, starting from 1:1 ratio.

Clearly, P9_B1 ${ }^{\mathrm{SPB}}$ was winning over P9_B1, regardless of the fluorescent marker used (Student's $t$-test, $\mathrm{P}=$

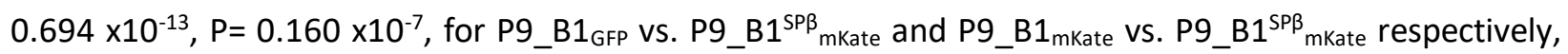
see methods) (Fig. 4C, Suppl. Fig. 4A). To confirm that competitive advantage of P9_B1 ${ }^{\text {SPB }}$ was due to pBGC (sublancin), we deleted the entire pBGC (sublancin production as well as immunity gene) or only the core gene sunA in P9_B1 ${ }^{\mathrm{SP} \beta}$ and challenged the mutants against the P9_B1 or P9_B1 ${ }^{\mathrm{SP} \beta}$. Both $\triangle \mathrm{pBGC}$ and $\Delta$ sunA strains lost their competitive advantage over the P9_B1 (Fig. 4C, Suppl. Fig. 4AB). In addition, $\Delta$ pBGC, but not $\Delta$ sunA was now loosing against P9_B1 ${ }^{\mathrm{SPB}}$ most likely due to lack of immunity to sublancin (Student's $t$ test, $\mathrm{P}=0.664 \times 10^{-10}, \mathrm{P}=0.301 \times 10^{-17}$, for P9_B1 ${ }^{\mathrm{SPB}}{ }_{\mathrm{GFP}}$ Vs. $\Delta \mathrm{PBGC}$ mKate and $\mathrm{P} 9 \_\mathrm{B}^{\mathrm{SPB}}{ }_{\text {mKate }} \mathrm{Vs} . \Delta \mathrm{pBGC} \mathrm{GFP}_{\mathrm{G}}$ respectively, see methods) (Fig. 4C, Suppl. Fig. 4B). As a control for potential fitness effects of the fluorescent markers, the co-cultures of isogenic strains were also examined, but the ratios remained as inoculated (Suppl. Fig. 4C). 
228 We took an alternative approach to examine competitive advantage from carrying an attachment site

229 (att) for pBGC phage on the chromosome. As not all B. subtilis strains carry $s p s M^{28}$, the lack of SP $\beta$

230 attachment gene would greatly reduce the frequency of SP $\beta$ integration into the host chromosome (Fig.

$2315 \mathrm{~A})$, and in effect, render the spsM-mutant less competitively fit compared to the wild type. We designed

232 a subsequent inoculation-based competition assay with P9_B1 vs. $\Delta s p s M$ in absence of SP $\beta$ (control) and

233 with a single episode of exposure to SP $\beta$ (see methods) (Fig. 5B). Both strains showed similar susceptibility

234 to lysis by SP $\beta$, based on growth curves and plaque assay (Suppl. Fig. 5A).

235 In the absence of the phage, both strains could co-exist at comparable relative proportions for at least 4

236 rounds of cultivation (see methods) (Fig. 5A, Suppl. Fig. 5B). However, a single exposure to SP $\beta$ at the

237 beginning of the experiment dramatically changed the competition outcome, resulting in higher

238 frequency of the P9_B1, regardless of fluorescent reporter used $\left(p<0.125 \times 10^{-3}, p<0.423 \times 10^{-3}\right.$ for $P 9$ _B $1_{\text {GFP }}$

239 vs. $\Delta s p s M_{\text {mKate }}$ and P9_B1 mKate Vs. $\Delta s p s M_{\text {GFP }}$ respectively, see methods) (Fig. 5A, Suppl. Fig. 5B). To test

240 whether the P9_B1 was winning due to higher rate of lysogenic conversion and pBGC acquisition, the

241 strains were separated to single colonies and tested their antagonistic activity against the P9_B1 ancestor

242 (Fig. 5B). Indeed, the vast majority of P9_B1 colonies were antagonistic against the ancestor, which was

243 not the case for $\Delta s p s M$. Finally, PCR screening of 4 randomly selected colonies from the $\Delta s p s M$ samples

244 confirmed that the presence of antagonistic activity correlated with integration of SP $\beta$-specific regions

245 (including sublancin) into the chromosome (Suppl. Fig. 5C). Using Nanopore sequencing, SP $\beta$ integration

246 was confirmed in an alternative locus $(y x z L)$ in absence of main att site in the bacterial chromosome

247 (Suppl. Fig. 6B).

248 These results demonstrated that a phages-borne pBGC confers a fitness benefit to the host bacterium, in

249 turn provokes a fitness benefit for the phage spreading as well. In addition, bacteria benefit from carrying

250 attachment sites in their genomes where pBGC-carrying phages can integrate. 
253 We then assessed the role of phages in horizontal gene transfer (HGT) of BGCs between closely and more 254 distantly related bacteria. First, all pBGCs prophages and virions along with their corresponding pBGCs 255 core genes were compared by average nucleotide identities. This allowed assessment of pBGC 256 conservation level within host genera, as well as the potential of phages to transfer pBGCs across genera 257 and family. When comparing core pBGC genes by average nucleotide identity, clustering according to 258 bacterial host genera was evident (Fig. 6, Suppl. Fig. 7). Most pBGC carrying virions retrieved from the PATRIC database clustered according to their PATRIC-annotated hosts and with ProphET-identified 260 prophages found in the corresponding host genus (Fig. 6, Suppl. Fig. 7). This confirms presence of a 261 functional lytic cycle in some pBGC-carrying prophages targeting Escherichia, Mainhemia, Listeria and 262 Lactobacillus opening up the possibility of pBGCs transfer via phage infection in nature.

263 Comparing the sequence similarity of $\mathrm{pBGC}$ carrying phages with the similarity of core pBGC genes, we 264 could identify 4 distinct patterns: 1) phage relatedness matches pBGCs relatedness; 2) similar pBGCs can 265 be found on distinct phages; 3) distinct pBGCs can be found on similar phages; 4) pBGC can be shared 266 across genera. First group is represented by Bacillus, Lactobacillus, or Listeria, where pBGCs carrying 267 phages as well as the pBGC core genes clearly clustered together (Fig. 6, Suppl. Fig. 7). For Listeria, the 268 pBGCs carrying phages, and pBGC core genes are nearly identical within the genus. Although the 269 remaining genera (Bacillus or Lactobacillus) contain some outlier pBGCs, which do not belong to the main 270 pBGC clusters, these were always associated with outlier prophages found within the corresponding 271 genera. This suggests that in Bacillus, Lactobacillus and Listeria, potential horizontal gene transfer of pBGC 272 may be restricted to the genus level. 
273 The second group is represented by Mainhemia, where all pBGCs are identical within genus, but they can

274 be found on diverse prophage elements (Fig. 6, Suppl. Fig. 7). This either indicates that pBGCs of

275 Mainhemia undergo between-phage horizontal gene transfer, or that they remain under purifying

276 selection-for instance due to their beneficial function for the bacterial host (Fig. 6, Suppl. Fig. 7).

277 The third group is represented by Enterococcus, where prophage diversity is rather limited (Fig. 6, Suppl.

278 Fig. 7) but the pBGC core genes are clearly divided into two different clusters (Fig. 6, Suppl. Fig. 7). Similar

279 phenomena can be observed even across different genera, like Mainhemia, Pasteurella and Heamophilus,

280 where the latter two carry Mainhemia-like prophages, but the pBGCs core genes remain unique for each

281 genus (Fig. 6). The same is the case for Aeromonas and Klebsiella, which carry Escherichia-like prophages,

282 but not Escherichia-like pBGCs core genes (Fig. 6), Suppl. Fig. 7. This may indicate that pBGCs play a role

283 in early diversification of phages towards different host species/genera. BLAST analysis confirmed that

284 most pBGCs (290 out of 307) had a match (>70 \% id, >70 \% length) within genome of another pBGC carrier

285 and only 17 were unique pBGCs as also observed from ANI plots (Fig. 6AB, Suppl. Fig. 7).

286 BLAST comparison also revealed that 19 pBGC carriers hosted an additional copy of a highly similar (80-

$28790 \%$ ) bacteriocin outside the original prophage region (Suppl. dataset 3). We also found that $5.1 \%$ of

288 genomes which were originally identified as non-pBGC carriers (14877 in total Fig. 1B) had at least one

289 analog to the pBGC core genes: in $56.4 \%$ of these, pBGC analogues were found in the same species as the

290 original pBGC host, $38.0 \%$ homologues were found in different species within the same genus as the pBGC

291 host, while $7.3 \%$ were found in different genera, but the same family (e.g. Escherichia > Shigella,

292 Manheimia > Glaesserella).

293 These results indicate that despite promiscuity of certain pBGCs carrying phages, and high potential for

294 cross-genus transfer of pBGCs, pBGCs core genes are rarely shared between different host genera. 


\section{Discussion}

297 Specialized metabolites play an important role in microbial interactions and are exploited by the

298 pharmaceutical industry, however, understanding the role and purpose of these compounds in nature has

299 only recently become an area of research. Here, we explored a novel concept suggesting that phages may

300 function as vectors for transferring biosynthetic potential for specialized metabolites therefore

301 contributing to their natural distribution and ecological impact. Although BGCs are rarely found within

302 phage genomes, when they are, they mostly reside within temperate phages or prophages.

303 Our bioinformatics approach has several drawbacks inherent in the scale of our analysis. ProphET

304 algorithm ${ }^{37}$ was used for prophage recognition as it is currently the best-performing stand-alone prophage

305 finder, although the reliance on a prophage database may underestimate novel prophages. AntiSMASH ${ }^{38}$

306 is currently the gold standard for profiling genomes for all types of BGCs, including genes encoding

307 bacteriocins.

308 Considering the relatively high abundance of other phage-encoded secreted compounds (e.g. toxins and

309 other virulence factors) which impact bacterial ecology $25,39,40$, one could expect phages to carry BGCs

310 encoding for bacteriocins. On the other hand, hosting large multi-gene assemblies like BGCs is probably

311 constrained by the efficiency of the phage DNA packing process, which translates to virion stability ${ }^{41}$. Such

312 size constrains could also explain why phages accommodate the smallest BGCs - those encoding

313 bacteriocins, which rarely exceed $10 \mathrm{~kb}^{42}$.

314 It was previously shown that host-associated lifestyle tends to correlate with higher prophage cargo ${ }^{43}$,

315 which is in line with our findings on higher abundance of pBGCs in commensal/pathogenic species (Fig.

$3162 A)$. 
317 Host lifestyle cannot be a sole predictor for pBGC, since some of the recurrent facultative pathogens e.g. Acinetobacter, Burkholderia, Pseudomonas or Staphylococcus, contained no, or very few pBGCs, despite presence of prophages ${ }^{44-46}$ In addition, in the vast majority of polylysogens (strains infected with multiple phages) containing even up to 19 prophages (Suppl. dataset 2), there was only only pBGC-carrying prophage. This might be simply due to immunity of the pBGC-carrier to infection by another pBGC-carrying phage, since pBGC-carriers targeting the same species are likely phylogenetically related.

The fact that $\mathrm{pBGCs}$ were underrepresented in bacteria with high numbers of BGCs in the genome (gBGCs), could suggest that the presence of gBGC reduces the likelihood of phage infections, matching previous data on role of gBGC in anti-phage defense ${ }^{47}$. Alternatively, lysogeny could reduce the likelihood of gBGC acquisition, for instance by negatively influencing genetic competence for transformation ${ }^{48,49}$. Nonetheless, our results are in line with BGCs-survey within human microbiome project, where the leading pBGCs-carriers Escherichia, Lactobacillus, Haemophilus and Enterococcus, were identified to have lowest numbers of BGCs (less than 2$)^{15}$.

Our work supports previous findings on temperate phages as carriers of putative weapons in interbacterial competition ${ }^{26}$. Previously, it was demonstrated that phages can repair BGCs by transducing missing genes between the strains ${ }^{50}$. Here, a complete and functional BGC is gained by prophage integration. Although the prophage itself (without pBGC) could serve as a biological weapon through spontaneous induction of lytic cycle in a subpopulation of cells ${ }^{51}$, this work demonstrates, in line with previous findings, that sublancin itself is sufficient to explain fitness benefits of lysogeny. The host also benefits from the intact phage attachment gene in presence of the $\mathrm{pBGC}$ phage. The sps $M$ gene has previously been shown to be involved in biofilm production and sporulation, indicating that the absence of $\mathrm{pBGC}$ prophage can also serve competitive advantage under certain ecological conditions e.g. in the absence of close relatives, susceptible to sublancin attack ${ }^{52,53}$. Here, the spsM locus provides function- 
independent benefits, uncovering fitness gain from certain chromosomal genes to capture pBGC phages more efficiently.

Recently, so called MuF polymorphic toxins have been predicted within multiple prophages of Firmicutes and proposed to serve as weapons in microbial warfare ${ }^{26}$. We see only minimal overlap between MuF toxin-carriers and pBGC-carrying phages from our dataset ( 9 bacterial genomes and only 5 prophages overlap) (compare Suppl. dataset 1 and Dataset $S 6^{26}$ ), confirming differences in genetic architecture, but not excluding similar ecological function, which in case of MuF toxins requires experimental validation. It

347 is also important to distinguish between phage-encoded bacteriocins and well-described virulence factors 348 residing in prophage regions e.g. the Shiga toxin encoded by E. coli lambdoid prophages, cholera toxin carried by Vibrio cholerae CTX phage or diphtheria toxin in Corynebacterium diphtheriae prophage, which target eukaryotic cells and may even hinder the inter-bacterial competition ${ }^{54}$. Discovering that nearly all pBGCs are bacteriocins also creates a fascinating connection to tailocins; bacterocidal phage tail-like particles, produced by certain genera, e.g. Pseudomonas, Vibrio, Burkholderia or Clostridium ${ }^{56}$. Although tailocin-carriers do not overlap with pBGC-carrying species, they probably share similar ecological role.

354 We observed that pBGC core genes match host phylogeny more than the entire phage genomes, which is 355 in line with previous results comparing phage genome vs single protein networks with host phylogeny ${ }^{57}$. 356 Previous work has revealed that the best phylogeny predictors are proteins that play a role in host 357 recognition ${ }^{57}$. Could bacteriocins be a good predictor of host range and what is the potential mechanism? 358 As phage specificity depends on recognition of specific receptors on the surface of host cells, by so called receptor binding proteins (RBPs), phage-encoded bacteriocins could recognize the same receptors. In fact, 360 PTS-glucose specific transport system, a putative sublancin target ${ }^{32}$ is also known to play a role in phage entry in E.coli ${ }^{58}$. 
362 Finally, clustering of pBGCs according to genus, combined with their role in interference competition,

363 could also point towards their role in bacterial speciation. The first evidence for this hypothesis has

364 recently begun to emerge from analysis of closely related, strains ${ }^{59-61}$.

365 Recent work on virome transplants indicate the tremendous role of phages in human health. It remains

366 to be discovered to what extent this can be assigned to pBGCs, likely due to fitness effects, an easy path

367 of HGT of those BGCs. Finally, prophage activation may occur during antibiotic therapy or even due to diet

368 choices $^{63}$, promoting spread of pBGCs across closely-related strains. Phages represent a treasure chest of

369 new BGCs with new activities. Here, we demonstrated the natural role of these phage-encoded BGCs in

370 interbacterial warfare and prove the plasticity of their transfer to new bacterial host, which can take us

371 forward in application of specialized metabolites. In summary, encoding of bacteriocins could be an

372 evolutionary strategy adapted by some phages to weaponize their host, thereby conferring a competitive

373 advantage of both the host and hence the phage infecting it. 


\section{Materials and methods}

\section{Strains and growth conditions}

377 All bacterial strains used in this study as well as strains used as genomic DNA donors are described in

378 Supplementary table 1 . Strains were routinely maintained in lysogeny broth (LB) medium (LB-Lennox, Carl

379 Roth; $10 \mathrm{~g} \mathrm{l}^{-1}$ tryptone, $5 \mathrm{~g} \mathrm{l}^{-1}$ yeast extract, and $5 \mathrm{~g} \mathrm{l}^{-1} \mathrm{NaCl}$ ). Strain DTUB231 (P9_B1 ${ }^{\mathrm{SP} \beta}$ ) was obtained by

380 infecting P9_B1 with a Bacillus phage SP $\beta$ isolated from strain MB8_B7. Isogenic lysogeny was confirmed

381 by colony PCR using primers pairs TB122/oAD2 (Supp. table 1) and oAD28/oAD3 (Supp. table 1) binding

382 to flanking prophage regions and host chromosome close to attL or attR, respectively. Colonies that were

383 positive for oTB122/OAD2 and oAD28/oAD3, but negative for oAD2/oAD3 (intact att) were selected as

384 stable SP $\beta$ lysogens. Strain DTUB208 was obtained by transforming MB8_B7 with gDNA of SPmini. DTUB43

385 and DTUB222 were obtained by transforming P9_B1 with phy_sGFP and phy_mKATE2 plasmids,

386 respectively. Strains DTUB235 and DTUB236 emerged from infecting of DTUB43 and DTUB222 with SP $\beta$

387 phage, respectively. Stable lysogens were confirmed by colony PCR like described above. Strains DTUB233

388 and DTUB234 were obtained by transforming DTUB43 and DTUB222 with gDNA isolated from GM3248

389 and selecting for kanamycin-resistant colonies. Strains DTUB244 and DTUB245 were obtained by

390 transforming DTUB43 and DTUB222 with gDNA isolated from $\triangle$ sunA and selecting for kanamycin-resistant

391 colonies, while DTUB246 and DTUB247 were obtained by transforming DTUB43 and DTUB222 with gDNA

392 isolated from ANC3, also selecting for kanamycin resistance. Deletions were confirmed using

393 oAD61/oAD62 (Supp. table 1) binding to sunA-flanking regions. Working concentrations of antibiotics

394 were $5 \mu \mathrm{g} \mathrm{ml}^{-1}$ for erythromycin, kanamycin and chloramphenicol, and $100 \mathrm{gg} \mathrm{m}^{-1}$ for spectinomycin.

395 Primers 27F/1492R (Supp. table 1) targeting 16S rRNA were used as a positive PCR control. 
Strain MB8_B7 was cultivated at $37^{\circ} \mathrm{C}$ with shaking at 200 r.p.m. In mid-exponential phase Mitomycin subsequently propagated in soft agar and liquid host suspension until the titer reached at least $10^{9}$ $\mathrm{pfu} / \mathrm{ml}$. Such culture supernatants were collected, adjusted to $\mathrm{pH}$ of 7.0, filter-sterilized and mixed at the solutions were centrifuged for $60 \mathrm{~min}$ at $12000 \mathrm{rpm}$ to obtain phage precipitates. The pellets were resuspended in $1 \%$ of the initial volume in SM buffer $\left(5.8 \mathrm{~g} \mathrm{l}^{-1} \mathrm{NaCl}, 0.96 \mathrm{~g} \mathrm{l}^{-1} \mathrm{MgSO}_{4}, 6 \mathrm{~g} \mathrm{l}^{-1} \mathrm{Tris}-\mathrm{HCl}, \mathrm{pH}\right.$ 7.5) to obtain concentrated solution of phage particles.

\section{Sublancin activity assay}

413 Sublancin activity assays were performed as previously described ${ }^{34}$. Briefly, overnight cultures of selected

414 strains were diluted $100 \times$, and $100 \mu$ of the diluted cultures were transferred onto the LB-agar (1.5\%)

415 plate using a plastic spreader to serve as a lawn (target strain). For the focal strains, undiluted overnight 416 cultures were spotted $(5 \mu \mathrm{l})$ on top of the lawn. Plates were incubated at $37^{\circ} \mathrm{C}$ for $24 \mathrm{~h}$. Clearing zone 417 around the focal colonies indicated their antimicrobial activity towards the target strain. 
420 Prior to competition assays the potential fitness costs of introducing fluorescent reporters, were

421 examined and it was noted that mKate-labelled strains may have a slight disadvantage in competition

422 (Suppl. Fig. 3). Therefore, all competition assays were performed with controls, where fluorescent

423 reporters were swapped. As the increase in GFP and mKate fluorescence in monoculture matched the

424 growth pattern of corresponding strains (Suppl., Fig.3), they were used as relative strain ratio indicators

425 in co-cultures. In co-cultures compensation correction was applied, to subtract the effect of GFP

426 background (0.044 \%) in mKate channel.

427 Overnight cultures of selected strains were obtained, optical density was measured and cultures were 428 pelleted down $(8000 \mathrm{~g}, 5 \mathrm{~min})$ and re-suspended in $0.9 \% \mathrm{NaCl}$, to reach optical density of 5 . Next, 1:1 co429 cultures were created by mixing equal volumes of selected strains suspensions. Such co-cultures were then inoculated at $1 \%$ into $200 \mu \mathrm{l}$ of LB distributed in 96-well microtiter plates. Cultivation was performed

431 in Synergy XHT multi-mode reader (Biotek Instruments, Winooski, VT, US), at $37^{\circ} \mathrm{C}$ with linear continuous 432 shaking ( $3 \mathrm{~mm})$, monitoring the optical density (600 $\mathrm{nm}$ ) as well as GFP (Ex: 482/20; Em:528/20; Gain: 35$)$ 433 and mKate (Ex: 590/20; Em: 635/32; Gain: 35) fluorescence every 10 min. For long-term competition 434 assay, co-cultures were transferred to fresh LB medium (2.5\% inoculum), every $24 \mathrm{~h}$.

435 Growth rates were calculated by linear regression on log transformed OD-values in exponential phase.

\section{Sample Preparation and LC-MS Analysis of Liquid Cultures}

438 To a $15 \mathrm{~mL}$ falcon tube magnesium sulfate $(280 \mathrm{mg})$ and sodium chloride $(140 \mathrm{mg})$ was added. Acidified

439 ( $0.1 \%$ trifluoroacetic acid) acetonitrile $(7 \mathrm{ml})$ was added, and shaken vigorously by hand for a short time. 440 Culture media $(7 \mathrm{ml})$ was then added to the solution. The mixture was extracted by shaking for $30 \mathrm{~min}$ at 441 room temperature. The sample was centrifuged (4200 CFU, $5 \mathrm{~min}$ ) to allow two liquid phases to form, an 442 organic, acetonitrile-rich, upper phase, and an aqueous, salt-rich, lower phase. The aqueous phase was 
443 removed and partially evaporated to approximately $5.5 \mathrm{ml}$. The partially evaporated aqueous phase was

444 then separated by solid phase extract (SPE), as follows: the aqueous extract was loaded onto a reversed-

445 phase SPE cartridge (Phenomenex Strata-X: $3 \mathrm{ml}, 30 \mathrm{mg}$ ) that had previously been conditioned with

446 methanol $(3 \mathrm{ml})$ and equilibrated with $0.1 \%$ formic acid in $\mathrm{H}_{2} \mathrm{O}(2 \mathrm{ml})$. After loading the sample onto the

447 column, the column was washed with acidified water $(0.1 \%$ formic acid, $2 \mathrm{ml})$, and the analytes were

448 eluted with 20,40 , and $80 \%$ (v/v) acetonitrile containing $0.1 \%$ formic acid (v/v) (1 ml fractions). Each of

449 the SPE eluents were transferred to individual HPLC vials for analysis by LC-MS using $15 \mu$ injection

450 volumes. LC-MS analysis was undertaken on a UHPLC-QTOF system, using gradient elution with reversed

451 phase chromatography. Refer to Supplementary methods for complete details on chemicals and LC-MS

452 conditions. Sublancin chemical features were detected in the $40 \%$ methanol fractions as $[\mathrm{M}+3 \mathrm{H}]^{3+}$ and

$453[\mathrm{M}+4 \mathrm{H}]^{4+}$ pseudo-molecular ions, as well as a characteristic $\left[\mathrm{M}-\mathrm{C}_{6} \mathrm{O}_{5} \mathrm{H}_{10}+4 \mathrm{H}\right]^{4+}$ fragmentation ion. All

454 cultures were extracted and analyzed in triplicate.

455

456

\section{Transmission electron microscopy}

457 Before use, 400 mesh nickel grids with a 3-4 $\mathrm{nm}$ thick carbon film, CF400-Ni-UL EMS (Electron

458 Microscopy Sciences, Hatfield, PA, USA) were hydrophilized by $30 \mathrm{~s}$ of electric glow discharging. Next,

$4595 \mu$ of purified phage solutions were applied onto the grids and allowed to adsorb for 1 min. The grids

460 were rinsed 3 times on droplets of milliQ water and subjected to staining with $2 \%$ uranyl acetate.

461 Specifically, with a help of EM grid-grade tweezers, the grids were placed sequentially on droplets of

$4622 \%$ uranyl acetate solution for $10 \mathrm{~s}, 2 \mathrm{~s}$ and $20 \mathrm{~s}$. Excess uranyl acetate was wicked away using filter

463 paper and the grids were allowed to dry overnight and stored in a desiccator until analysis.

464 Transmission electron microscopy was performed utilizing a FEI Tecnai T12 Biotwin TEM (Thermo Fisher

465 Scientific, Hillsboro, OR, USA) operating at $120 \mathrm{kV}$ located at the Center for Electron Nanoscopy at the 
466 Technical University of Denmark, and images were acquired using a Bottom mounted CCD, Gatan Orius

467 SC1000WC (Gatan Inc., Pleasanton, CA, USA).

468

469

pBGC mining

470 All complete bacterial refseq genomes were downloaded using ncbi-genome-download

471 (https://github.com/kblin/ncbi-genome-download). Next, all genomes were profiled using a custom script

472 in which prophages were found by using ProphET ${ }^{37}$, in which blastp was substituted for DIAMOND for

473 performance reasons. Next, total genomic biosynthetic gene clusters (gBGCs) were found

474 using antiSMASH $4^{38}$ with the -minimal option enabled, again for performance reasons. Third, prophage

475 encoded BGCs (pBGCs) were found by rerunning antiSMASH4 on the predicted prophages. The complete

476 code is available on request.

477 Certain species are overrepresented in the genome database and many of the genomes are highly similar

478 which may give both additional false positives and negatives. Dereplication of $15,000+$ genomes was 479 considered unfeasible.

481 Profiling of pBGCs

482 pBGCs were annotated using blastp against the BACTIBASE database ${ }^{64}$ with default settings. Presence of 483 pBGC core genes outside the prophage regions were investigated by using blastn against the host and 484 non-host genomes with identity of $70 \%$ and a coverage of $50 \%$. 
487 Phages positive for pBGCs were further analyzed by average nucleotide identity using pyani

488 (https://github.com/widdowquinn/pyani), and the resulting nucleotide identity matrix was clustered by

489 hierarchical clustering using hclust() from $\mathrm{R}$ on the corresponding distance matrix.

490 A network of pBGCs was made by considering the ANI as an adjacency matrix using the igraph package in

$491 \quad$ R and building a weighted and directed graph.

492 The Phaster software ${ }^{65,66}$ was used to detect prophage elements in natural B. subtilis isolates. Next, the 493 sequence of SP $\beta$-like prophage detected in MB8_B7 was compared with SP $\beta$ sequence of $B$. subtilis 168

494 using blast. Lack of ICEBs1 (or its fragments) in MB8_B7 was confirmed by blast.

\section{Whole genome sequencing by MiniON Nanopore}

Bacterial genomic DNA was isolated using EURex Bacterial and Yeast Genomic DNA Kit, while phage DNA was extracted using the phenol:chloroform method as described previously ${ }^{67}$. The qualities of the extracted gDNA were evaluated by NanoDrop DS-11+ Spectrophotometer (Saveen Werner, Sweden) and electrophoresis, then quantified with the High Sensitivity DNA kit and Qubit 2.0 Fluorometer (Thermo Fisher Scientific). For multiplex MiniON sequencing, the Rapid Barcoding gDNA Sequencing kit was used

503 prepared using the SQK-RBK004 rapid barcoding kit and subsequently loaded into the MinION Flow Cell.

504 The local base calling was performed automatically in real-time using the MinKNOW ONT software

505 (v3.1.19). De-multiplexing and adapter trimming was carried out using EPI2ME Agent software 506 (v2020.2.10) using the Fastq Barcoding r2020.03.10 function and the genomes were then assembled using Unicycler v0.4.8 ${ }^{68}$. 
The association between pBGC positivity versus prophage or gBGC counts was tested for significance using Mann-Whitney U-tests.

511 To calculate the individual effects of the SP $\beta$ prophage, the intact $\mathrm{pBGC}$ or only the sunA gene on strain

512 performance during competition, the change in proportion from $0 \mathrm{~h}$ to $24 \mathrm{~h}$ in a strain with a given gene

513 content was compared to the change in proportion of the non-modified strain with the same fluorescent

514 marker. Statistical differences between two experimental groups were then identified using a two-tailed

515 Student's $t$-tests assuming equal variance. To assess the effect of phage att site (sps $M$ ) on competition in

516 the presence and absence of SP $\beta$, changes in P9_B1 proportion throughout the competition (start vs $3^{\text {rd }}$

517 at transfer $24 \mathrm{~h}$ ) in the absence of SP $\beta$, where compared to changes of its proportion during competition

518 with single exposure to SP $\beta$. Relative abundance of strains in co-culture was calculated based on

519 fluorescence values (see Suppl. Fig. 3). Normal distributions within the above datasets were confirmed by

520 Kolmogorov-Smirnov $(P>0.05)$. Differences in growth rates between P9_B1, P9_B1 $1_{\mathrm{GFP}}$ and P9_B1 mKate were

521 assessed by One-Way ANOVA and mean comparisons by Tukey test. No outliers were removed from the

522 dataset. No statistical methods were used to predetermine sample size and the experiments were not

523 randomized.

\section{Data availability}

525 Raw sequencing data have been deposited to SRA databased under SUB8228942 (for $\Delta$ spsM $^{\mathrm{SP} \beta}$ ) and 526 SUB8237787 (for SP $\beta$ ) accession numbers. The complete code used for mining of phage-encoded BGCs is 527 available on request from Mikael Lenz Strube. 
Authors contributions:

530

531

532

533

534

535

536

537

538

539

540

541

542

543

544

545

546

547

548

549

A.D. and M.L.S. designed the study. M.L.S. performed bioinformatic analysis, A.D. performed the experiments, A.J.C.A. performed chemical analysis, C.N.L.A. performed Nanopore sequencing and data processing, A.T.K. provided molecular tools for the study. A.D. and M.L.S. wrote the manuscript. All authors contributed to final version of the manuscript.

The authors declare no conflict of interest.

\section{Acknowledgements:}

The authors thank L. Gram for feedback during ongoing project and constructive comments on the final version of this manuscript. We also thank A. Kofod-Petersen for valuable discussions. This work was supported by the Danish National Research Foundation (DNRF137) for the Center for Microbial Secondary Metabolites.

\section{Figure Legends}

Fig. 1. Bioinformatic worflow used to extract phage- or prophage-encoded biosythetic gene clusters

(pBGCs). A. All high- quality phage genomes available in PATRIC database (10062) were screened by antiSMASH, and only $0.07 \%$ appeared to contain pBGCs. The vast majority of these pBGCs-containing phages (92.3\%) were capable of lysogenic lifestyle, as confirmed by literature mining. B. All high quality bacterial genomes available from NCBI (15184) were screened by ProphET to extract prophage rergions, which were next screened by antiSMASH to detect pBGCs. Relative abundance of pBGCs within prophages was nearly $3 \%$. 
Fig. 2: Distribution of pBGC across genera. A) Relative distribution of bacteria which serve as hosts to pBGC-carrying phages colored by genus. For colors interpretation, see figure legend in 2B. Pink indicates Shigella genus. B) Number of prophages in each genome versus number of genomic BGCs colored by genus. Genomes having only one $\mathrm{pBGC}$ are marked by triangles and genomes containing multiple pBGCs are marked by diamonds. Points have been jittered to avoid overlap.

Fig. 3: BLASTP identities and alignment lengths of pBGC core proteins. Points are colored by their closest match and have been jittered to avoid overlap. Histograms show densities for the corresponding blastp metric. is lysogenic for the SP $\beta$ prophage which carries a $\mathrm{pBGC}$ responsible for sublancin biosynthesis, sunA being the core gene. The SP $\beta$ prophage was induced and purified, as confirmed by transmission electron microscopy (scale bar $=100 \mathrm{~nm}$ ) and used to infect the soil isolate P9_B1 (pictured in green) resulting in P9_B1 ${ }^{\mathrm{SPB}}$ (pictured in brown). Antagonistic activity/immunity of $\mathrm{MB} 8 \_\mathrm{B} 7, \mathrm{P} 9 \_\mathrm{B} 1$ and $\mathrm{P9}$ - $\mathrm{B} 1^{\mathrm{SPB}}$ were indicated as square diagram, where square represents a lawn and dot represents a focal strain. A white ring around the focal strain indicates its antagonistic activity against the lawn strain. B. Detection of sublancin in supernatants of P9_B1, MB8_B7, MB8_B7 $\triangle \mathrm{SP} \beta$ and P9_B1 ${ }^{\mathrm{SPB}}$ using LC-MS. C. Competition

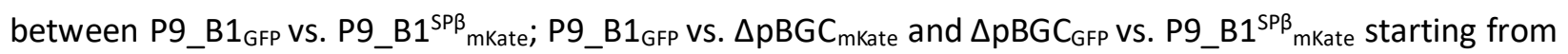
GFP and mKate fluorescence, shown in yellow and purple, respectively. Error bars (light grey) indicates standard error $(n=6)$.

571 typically integrates into spsM gene splitting this gene. It was hypothesized that frequency of lysogenic 572 conversion will be greatly reduced if spsM gene is removed. Below - long term competition assay between 
573 initially SP $\beta$-negative P9_B1 $1_{\text {mkate }}$ vs $\Delta$ spsM $M_{\text {GFP }}$ without and with single exposure to SP $\beta$ at the exponential

574 growth phase during first co-cultivation round. All assays were initiated at 1:1 proportions. Relative

575 abundance of two competing strains was monitored by changes in relative GFP and mKate fluorescence,

576 shown in yellow and purple, respectively. Error bars (light grey) indicate standard error $(n=8)$. B. Mixed

577 populations of P9_B1 and $\Delta$ spsM after long-term cultivation experiment were screened for antagonistic

578 activity against the P9_B1 ancestor. All co-cultures exposed to SP $\beta$ acquired antagonistic activity towards

579 the P9_B1, which was not observed in any of the control co-cultures (no phage solution added). P9_B1

580 and $\triangle s p s M$ were separated to single colonies and again screened for antagonistic activity against the

581 P9_B1 ancestor by striking the colonies on top of P9_B1 lawn. Plates were illuminated by UV lamp and

582 imaged by iPhone7 and the fraction of P9_B1 and $\triangle$ spsM colonies inhibitory towards P9_B1 was

583 calculated. Green strikes indicate GFP-labelled strains and pink strikes indicate mKate-labelled strains. ${ }^{* * *}$

584 indicates $\mathrm{P}<0.001(\mathrm{n}=4)$.

Fig. 6. Diversity of phages and pBGCs. A) Network of $\mathrm{pBGC}$ similarity of full length prophages and virions carrying pBGCs, labelled by major genera. Each edge is weighted by the ANI-value of each pair. B) Network of pBGC core gene similarity of prophage- and virion-derived pBGCs, labelled by major genera. Each edge is weighted by the ANI-value of each pair.

\section{References}

591 1. Belknap, K. C., Park, C. J., Barth, B. M. \& Andam, C. P. Genome mining of biosynthetic and

592 chemotherapeutic gene clusters in Streptomyces bacteria. Sci. Rep. 10, 1-9 (2020).

593 2. Hug, J. J., Krug, D. \& Müller, R. Bacteria as genetically programmable producers of bioactive $594 \quad$ natural products. Nature Reviews Chemistry vol. 4 172-193 (2020). 
3. Traxler, M. F. \& Kolter, R. Natural products in soil microbe interactions and evolution. Nat. Prod.

Rep. 32, 956-970 (2015).

597

4. Granato, E. T., Meiller-Legrand, T. A. \& Foster, K. R. The Evolution and ecology of bacterial warfare. Curr. Biol. 29, R521-R537 (2019).

5. Coyte, K. Z., Schluter, J. \& Foster, K. R. The ecology of the microbiome: Networks, competition, and stability. Science 350, 663-666 (2015).

6. Weissman, K. J. \& Leadlay, P. F. Combinatorial biosynthesis of reduced polyketides. Nature Reviews Microbiology vol. 3 925-936 (2005).

7. Süssmuth, R. D. \& Mainz, A. Nonribosomal Peptide Synthesis-principles and prospects. Angew.

8. Li, Y. \& Rebuffat, S. The manifold roles of microbial ribosomal peptide-based natural products in physiology and ecology. Journal of Biological Chemistry vol. 295 34-54 (2020).

9. Biggins, J. B., Ternei, M. A. \& Brady, S. F. Malleilactone, a polyketide synthase-derived virulence 608 factor encoded by the cryptic secondary metabolome of Burkholderia pseudomallei group pathogens. J. Am. Chem. Soc. 134, 13192-13195 (2012).

10. Quadri, L. E. N., Sello, J., Keating, T. A., Weinreb, P. H. \& Walsh, C. T. Identification of a Mycobacterium tuberculosis gene cluster encoding the biosynthetic enzymes for assembly of the virulence-conferring siderophore mycobactin. Chem. Biol. 5, 631-645 (1998).

613 11. Tyc, O., Song, C., Dickschat, J. S., Vos, M. \& Garbeva, P. The Ecological role of volatile and soluble secondary metabolites produced by soil bacteria. Trends in Microbiology vol. 25 280-292 (2017). 

(2019).

13. Davies, J., Spiegelman, G. B. \& Yim, G. The world of subinhibitory antibiotic concentrations. Curr. Opin. Microbiol. 9, 445-453 (2006).

14. Blin, K., Kim, H. U., Medema, M. H. \& Weber, T. Recent development of antiSMASH and other computational approaches to mine secondary metabolite biosynthetic gene clusters.

15. Donia, M. S. et al. A systematic analysis of biosynthetic gene clusters in the human microbiome reveals a common family of antibiotics. Cell 158, 1402-1414 (2014). doi:10.1093/bib/bbx146.

16. Sharrar, A. M. et al. Bacterial secondary metabolite biosynthetic potential in soil varies with

627 17. Libis, V. et al. Uncovering the biosynthetic potential of rare metagenomic DNA using coR859-R864 (2016).

19. Baümler, A. J. \& Sperandio, V. Interactions between the microbiota and pathogenic bacteria in the gut. Nature vol. 535 85-93 (2016).

20. Knowles, B. et al. Lytic to temperate switching of viral communities. Nature 531, 466-470 (2016).

21. Rasmussen, T. S. et al. Faecal virome transplantation decreases symptoms of type 2 diabetes and obesity in a murine model. Gut 0, 1-9 (2020). 
mechanisms, impact and ecology of tem perate phages. ISME J. 11, 1511-1520 (2017).

23. Casjens, S. Prophages and bacterial genomics: What have we learned so far? Molecular Microbiology vol. 49 277-300 (2003).

24. Boyd, E. F. Bacteriophage-encoded bacterial virulence factors and phage-pathogenicity island interactions. in Advances in Virus Research vol. 82 91-118 (Academic Press Inc., 2012).

25. Castillo, D. et al. Widespread distribution of prophage-encoded virulence factors in marine Vibrio communities. Sci. Rep. 8, 1-9 (2018).

26. Jamet, A. et al. A widespread family of polymorphic toxins encoded by temperate phages. $B M C$ Biol. 15, 75 (2017).

27. Hemphill, H. E., Gage, I., Zahler, S. A. \& Korman, R. Z. Prophage-mediated production of a bacteriocinlike substance by SP $\beta$ lysogens of Bacillus subtilis. Can. J. Microbiol. 26, 1328-1333 (1980).

28. Dragos, A. et al. Phage recombination drives evolution of spore-forming Bacilli. bioRxiv 2020.05.06.055103 (2020) doi:10.1101/2020.05.06.055103.

29. Garcia De Gonzalo, C. V., Zhu, L., Oman, T. J. \& Van Der Donk, W. A. NMR structure of the S-linked glycopeptide sublancin 168. ACS Chem. Biol. 9, 796-801 (2014).

30. Oman, T. J., Boettcher, J. M., Wang, H., Okalibe, X. N. \& van der Donk, W. A. Sublancin is not a 654 lantibiotic but an S-linked glycopeptide. Nat. Chem. Biol. 7, 78-80 (2011).

31. Dorenbos, R. et al. Thiol-disulfide oxidoreductases are essential for the production of the lantibiotic sublancin 168. J. Biol. Chem. 277, 16682-16688 (2002). 
658

659

660

661

662

663

664

665

666

667

668

669

670

671

672

673

674

675

676

677

678

involved in sensitivity to the glucosylated bacteriocin sublancin. Antimicrob. Agents Chemother.

59, 6844-6854 (2015).

33. Wu, C., Biswas, S., Garcia De Gonzalo, C. V. \& Van Der Donk, W. A. Investigations into the mechanism of action of sublancin. ACS Infect. Dis. 5, 454-459 (2019).

34. Dubois, J. Y. F. et al. Immunity to the bacteriocin sublancin 168 is determined by the Sunl (YolF) protein of Bacillus subtilis. Antimicrob. Agents Chemother. 53, 651-661 (2009).

35. Kiesewalter, H. T. et al. Complete genome sequences of 13 Bacillus subtilis soil isolates for studying secondary metabolite diversity . Microbiol. Resour. Announc. 9, (2020).

36. Eleina England, by M. \& Bell Professor of Biology, S. P. Effects of cell growth and a mobile genetic element on propagation of the phages SP16 and SP-beta in Bacillus subtilis (2014).

37. Reis-Cunha, J. L., Bartholomeu, D. C., Manson, A. L., Earl, A. M. \& Cerqueira, G. C. ProphET, prophage estimation tool: A standalone prophage sequence prediction tool with self-updating reference database. PLoS One 14, 1-9 (2019).

38. Blin, K. et al. AntiSMASH 4.0 - improvements in chemistry prediction and gene cluster boundary identification. Nucleic Acids Res. 45, W36-W41 (2017).

39. Wagner, P. L. \& Waldor, M. K. Bacteriophage control of bacterial virulence. Infection and Immunity vol. 70 3985-3993 (2002).

40. Jamet, A. et al. A widespread family of polymorphic toxins encoded by temperate phages. BMC Biol. 15, (2017).

41. Hatfull, G. F. \& Hendrix, R. W. Bacteriophages and their genomes. Curr. Opin. Virol. 1, 298-303

(2011). 
50. Matilla, M. A. \& Salmond, G. P. C. Bacteriophage $\varphi M A M 1$, a viunalikevirus, is a broad-host-range,

42. Maqueda, M. et al. Genetic features of circular bacteriocins produced by Gram-positive bacteria. FEMS Microbiol. Rev. 32, 2-22 (2008).

43. Touchon, M., Bernheim, A. \& Rocha, E. P. Genetic and life-history traits associated with the distribution of prophages in bacteria. ISME J. 10, 2744-2754 (2016).

44. Costa, A. R., Monteiro, R. \& Azeredo, J. Genomic analysis of Acinetobacter baumannii prophages reveals remarkable diversity and suggests profound impact on bacterial virulence and fitness. Sci. Rep. 8, 15346 (2018).

45. Roszniowski, B., McClean, S. \& Drulis-Kawa, Z. Burkholderia cenocepacia prophages-prevalence, chromosome location and major genes involved. Viruses 10, (2018).

46. Goerke, C. et al. Diversity of prophages in dominant Staphylococcus aureus clonal lineages. J. Bacteriol. 191, 3462-3468 (2009).

47. Kronheim, S. et al. A chemical defence against phage infection. Nature (2018) doi:10.1038/s41586-018-0767-x.

48. Brooks, M. R. et al. Prophage-mediated disruption of genetic competence in Staphylococcus pseudintermedius . mSystems 5, (2020).

49. Mashburn-Warren, L., Goodman, S. D., Federle, M. J. \& Prehna, G. The conserved mosaic prophage protein paratox inhibits the natural competence regulator ComR in Streptococcus. Sci. Rep. 8, 1-15 (2018). high-efficiency generalized transducer that infects environmental and clinical isolates of the enterobacterial genera Serratia and Kluyvera. Appl. Environ. Microbiol. 80, 6446-6457 (2014). 
700

701

702

703

704

705

706

707

708

709

710

711

712

713

714

51. Nanda, A. M., Thormann, K. \& Frunzke, J. Impact of spontaneous prophage induction on the fitness of bacterial populations and host-microbe interactions. Journal of Bacteriology vol. 197 410-419 (2015).

52. Sanchez-Vizuete, P. et al. Identification of ypqP as a new Bacillus subtilis biofilm determinant that mediates the protection of Staphylococcus aureus against antimicrobial agents in mixed-species communities. Appl. Environ. Microbiol. 81, 109-18 (2015).

53. Abe, K. et al. Developmentally-regulated excision of the SP $\beta$ prophage reconstitutes a gene required for spore envelope maturation in Bacillus subtilis . PLoS Genet. 10, e1004636 (2014).

54. Paquette, S. J., Zaheer, R., Stanford, K., Thomas, J. \& Reuter, T. Competition among Escherichia coli strains for space and resources. Vet. Sci. 5, (2018).

55. Wagner, P. L. et al. Bacteriophage control of Shiga toxin 1 production and release by Escherichia coli. Mol. Microbiol. 44, 957-970 (2002).

56. Ghequire, M. G. K. \& De Mot, R. The Tailocin Tale: peeling off phage tails. Trends in Microbiology vol. 23 587-590 (2015).

57. Shapiro, J. W. \& Putonti, C. Gene co-occurrence networks reflect bacteriophage ecology and evolution. MBio 9, (2018).

58. Esquinas-Rychen, M. \& Erni, B. Facilitation of bacteriophage lambda DNA injection by inner membrane proteins of the bacterial phosphoenol-pyruvate: Carbohydrate Phosphotransferase System (PTS) JMMB Symposium. J. Mol. Microbiol. Biotechnol vol. 3 (2001).

59. Stefanic, P., Kraigher, B., Lyons, N. A., Kolter, R. \& Mandic-Mulec, I. Kin discrimination between sympatric Bacillus subtilis isolates. Proc. Natl. Acad. Sci. 112, 14042-14047 (2015). 
$72160 . \quad$ Lyons, N. A., Kraigher, B., Stefanic, P., Mandic-Mulec, I. \& Kolter, R. A combinatorial kin discrimination system in Bacillus subtilis. Curr. Biol. 26, 733-742 (2016).

723

61. Song, S., Guo, Y., Kim, J.-S., Wang, X. \& Wood, T. K. Phages mediate bacterial self-recognition. Cell Rep. 27, 737-749.e4 (2019).

62. Czech, L. et al. Role of the extremolytes ectoine and hydroxyectoine as stress protectants and nutrients: Genetics, phylogenomics, biochemistry, and structural analysis. Genes vol. 9 (2018).

63. Boling, L. et al. Dietary prophage inducers and antimicrobials: toward landscaping the human gut microbiome. Gut Microbes 11, 721-734 (2020).

64. Hammami, R., Zouhir, A., Le Lay, C., Ben Hamida, J. \& Fliss, I. BACTIBASE second release: A database and tool platform for bacteriocin characterization. BMC Microbiol. 10, (2010).

65. Zhou, Y., Liang, Y., Lynch, K. H., Dennis, J. J. \& Wishart, D. S. PHAST: A Fast Phage Search Tool. Nucleic Acids Res. 39, (2011).

66. Arndt, D. et al. PHASTER: a better, faster version of the PHAST phage search tool. Nucleic Acids Res. 44, W16-W21 (2016).

67. Tóth, I., Sváb, D., Bálint, B., Brown-Jaque, M. \& Maróti, G. Comparative analysis of the Shiga toxin converting bacteriophage first detected in Shigella sonnei. Infect. Genet. Evol. 37, 150-157 (2016).

68. Wick, R. R., Judd, L. M., Gorrie, C. L. \& Holt, K. E. Unicycler: Resolving bacterial genome assemblies from short and long sequencing reads. PLoS Comput. Biol. 13, 1-22 (2017).

69. Westers, H. et al. Genome engineering reveals large dispensable regions in Bacillus subtilis. Mol. Biol. Evol. 20, 2076-2090 (2003). 
bioRxiv preprint doi: https://doi.org/10.1101/2020.10.01.322628; this version posted October 1,2020. The copyright holder for this preprint (which was not certified by peer review) is the author/funder, who has granted bioRxiv a license to display the preprint in perpetuity. It is made available under aCC-BY-ND 4.0 International license.

742 70. van Gestel, J., Weissing, F. J., Kuipers, O. P. \& Kovács, Á. T. Density of founder cells affects spatial pattern formation and cooperation in Bacillus subtilis biofilms. ISME J. 8, 2069-79 (2014).

744 71. Dragoš, A. et al. Division of labor during biofilm matrix production. Curr. Biol. 28, 1903-1913.e5 (2018). 
A. B.
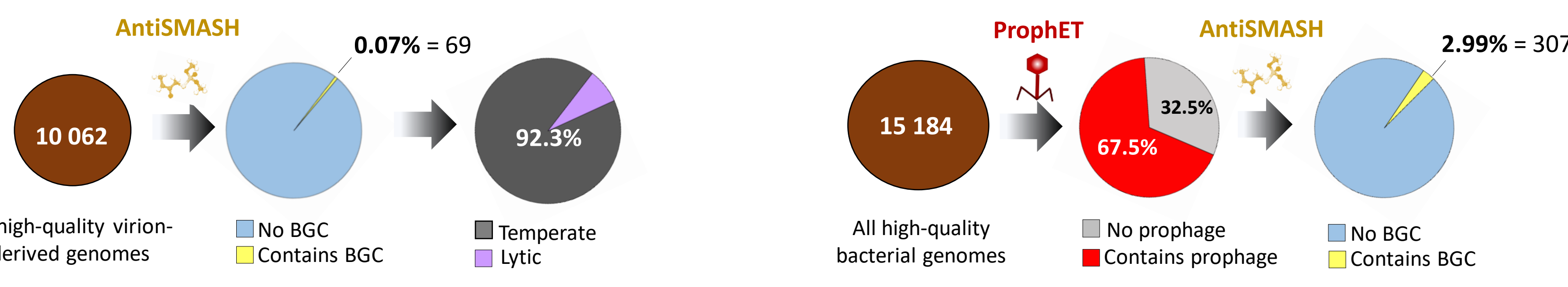
A.

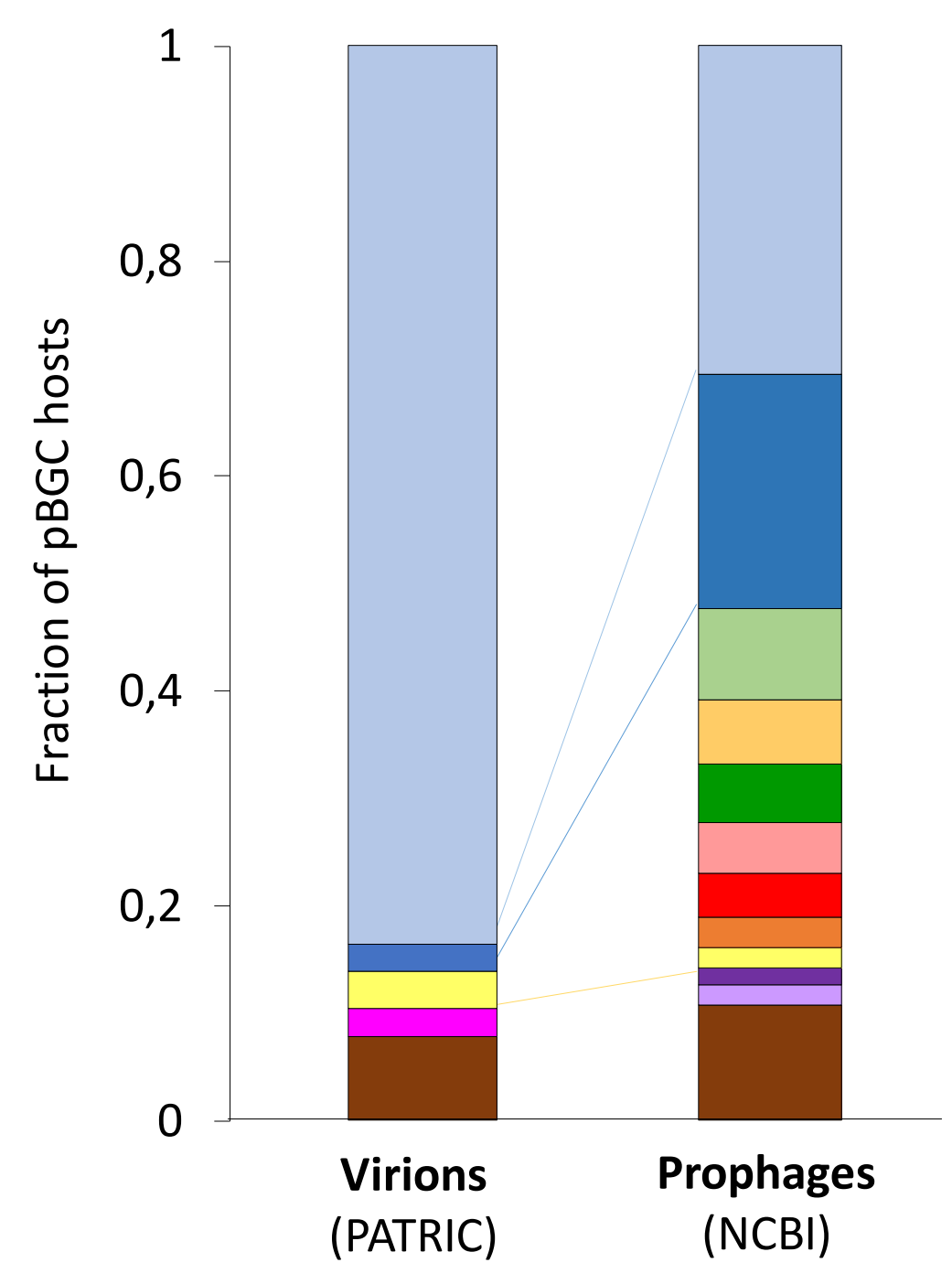

B.





A.

B.

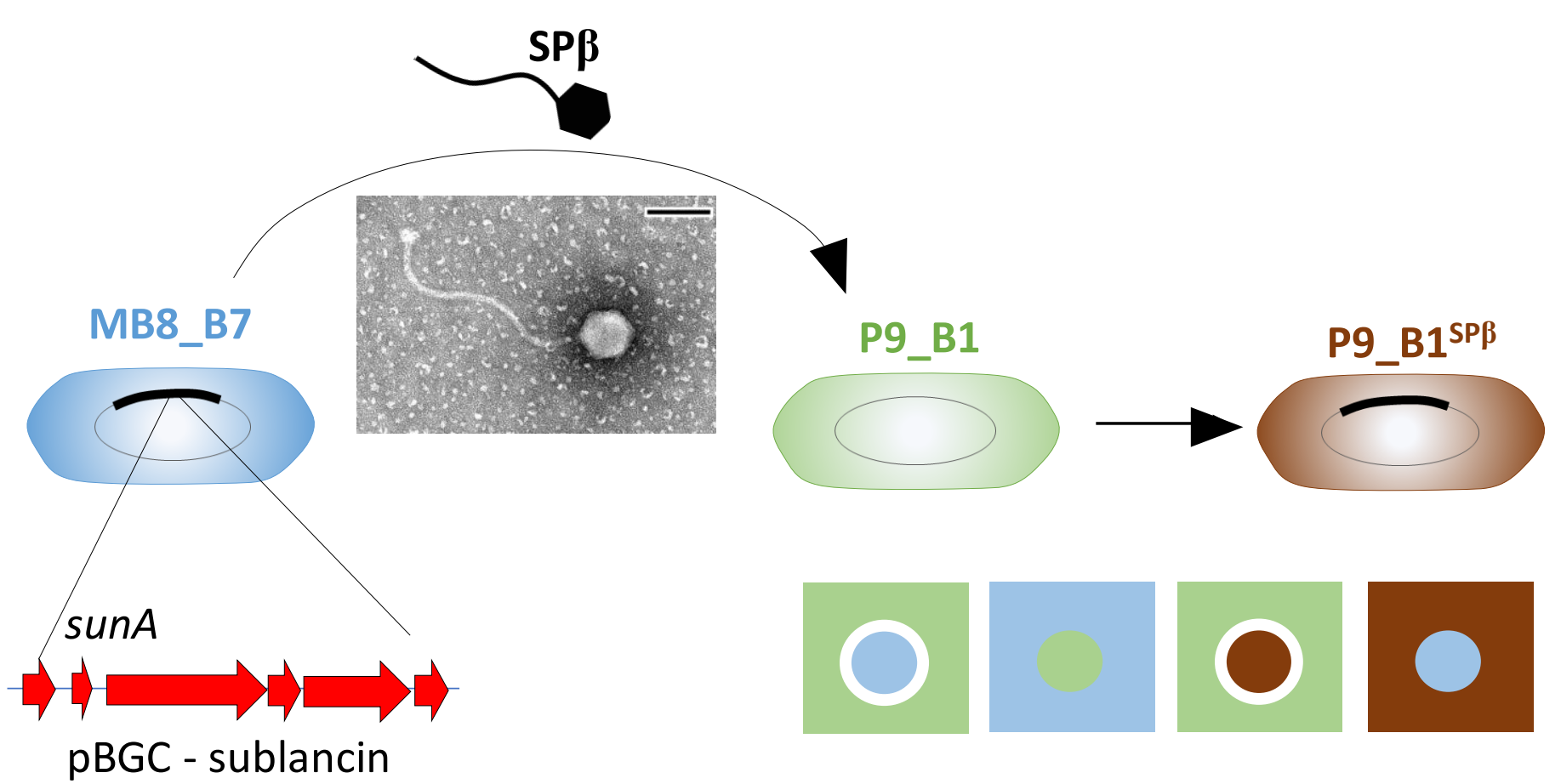

C.

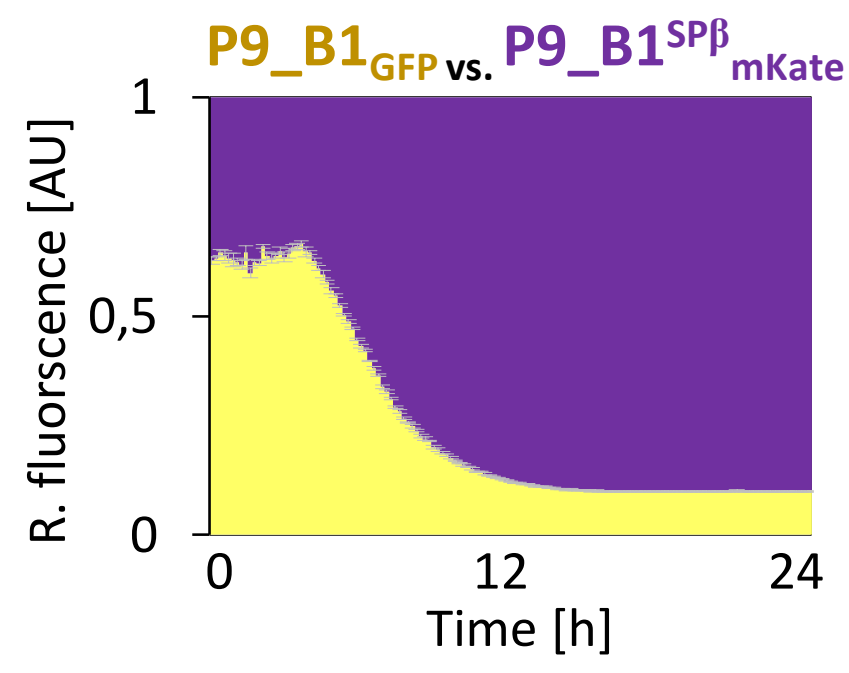

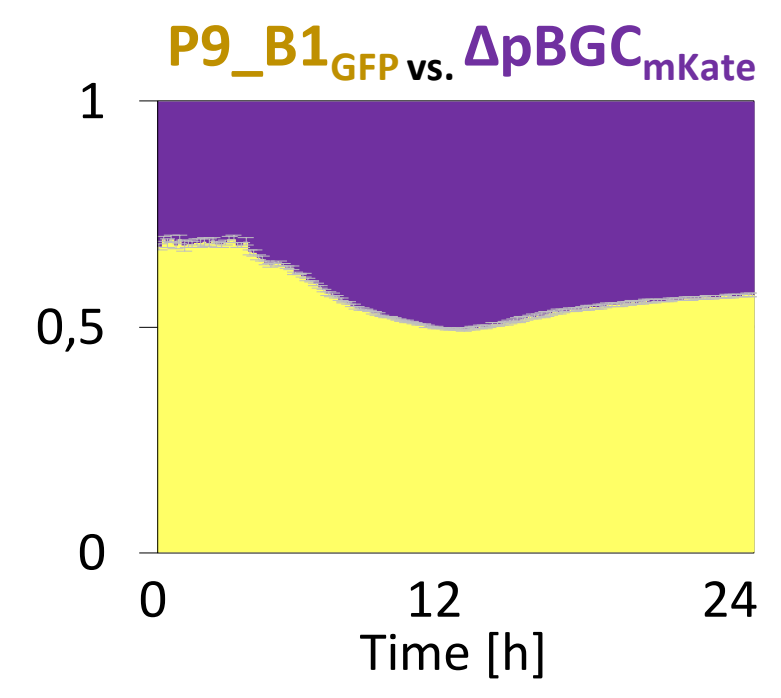

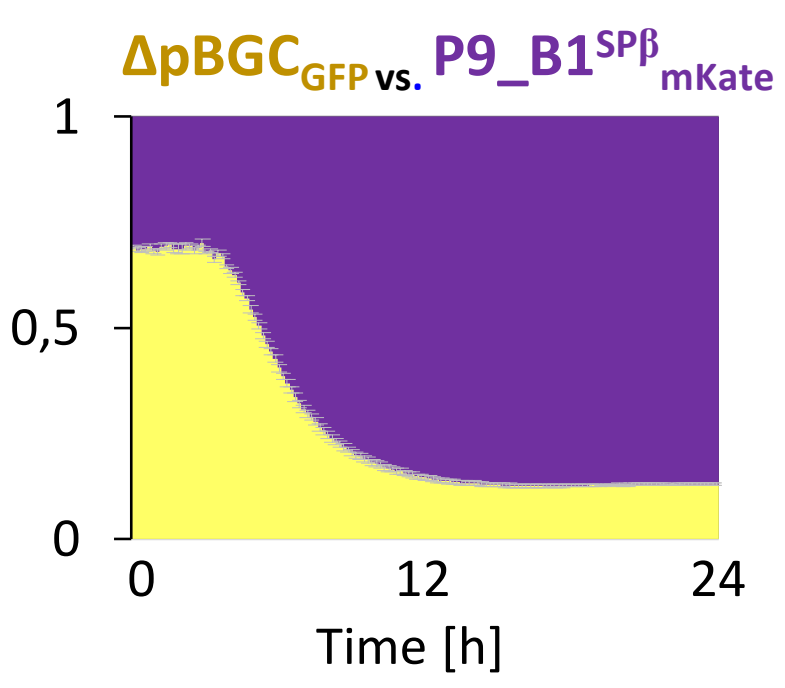

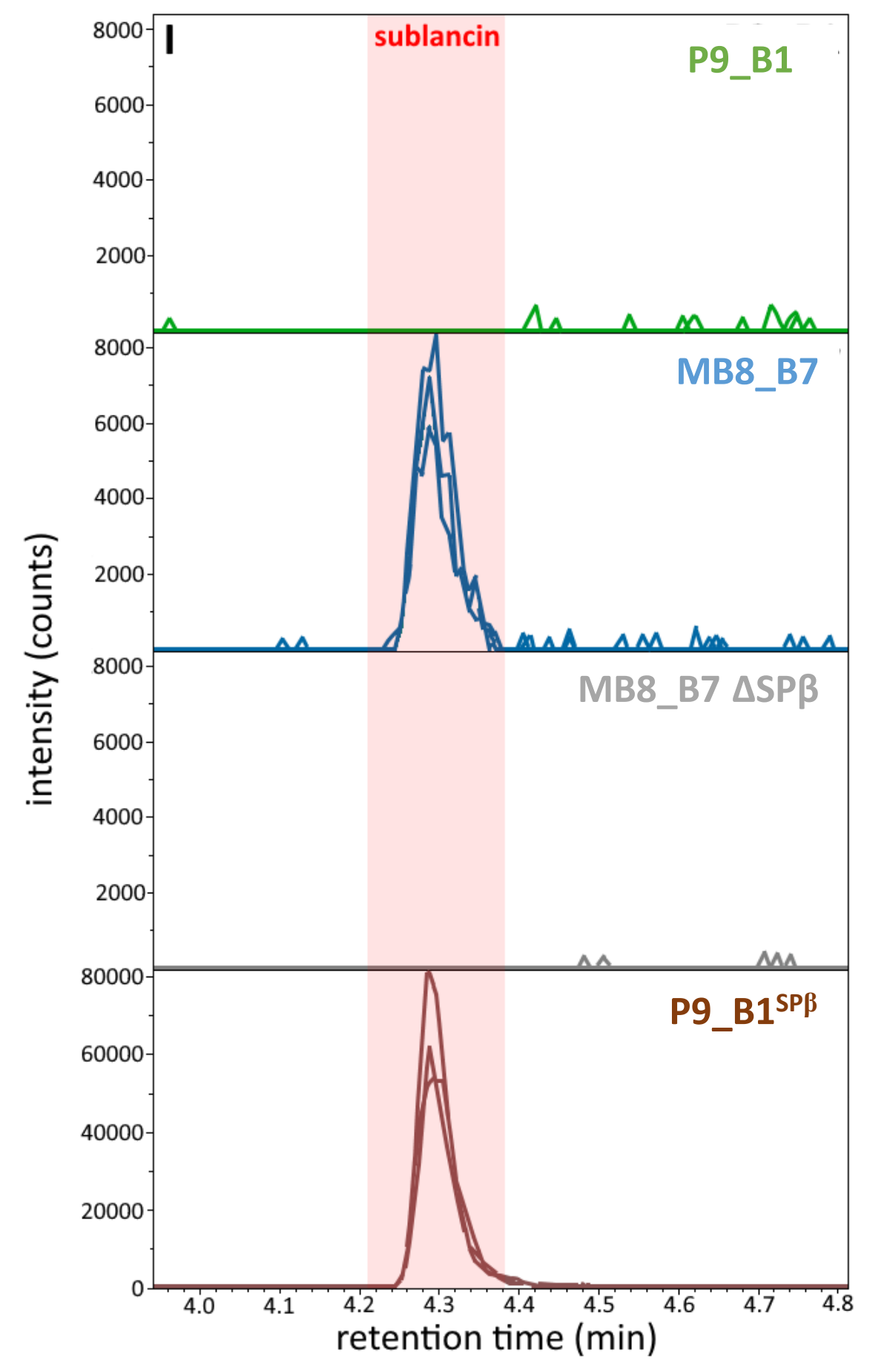

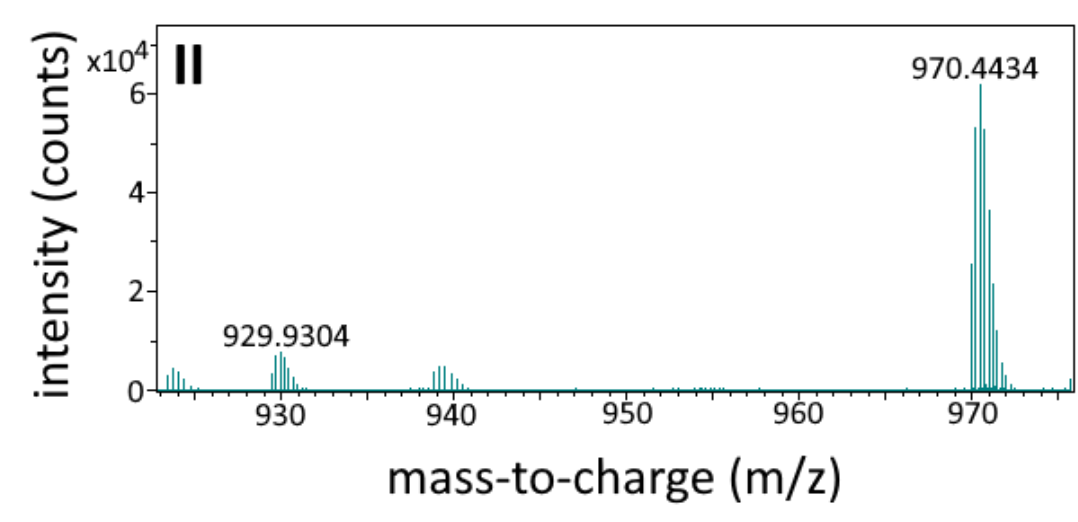


A.
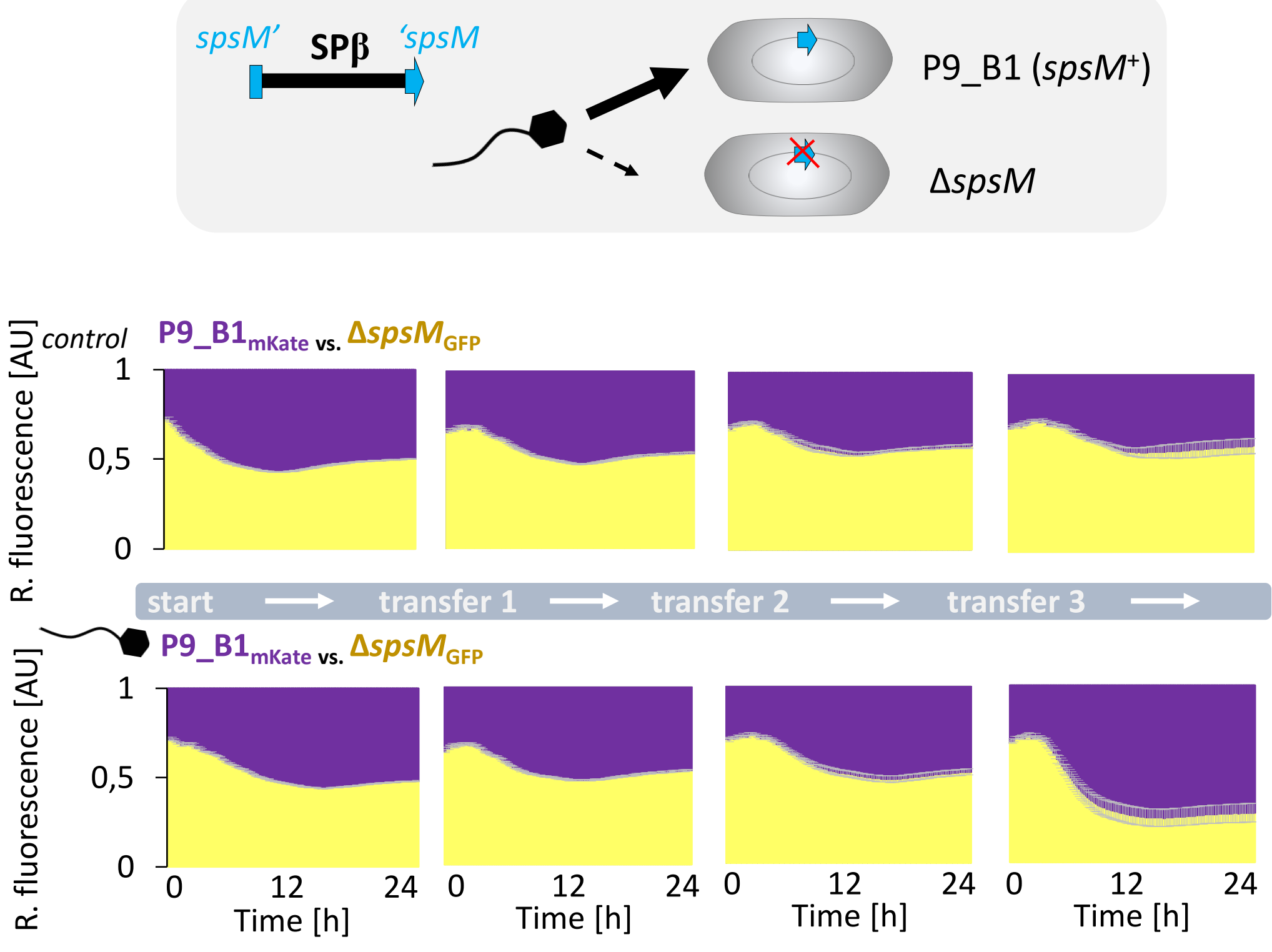

B.

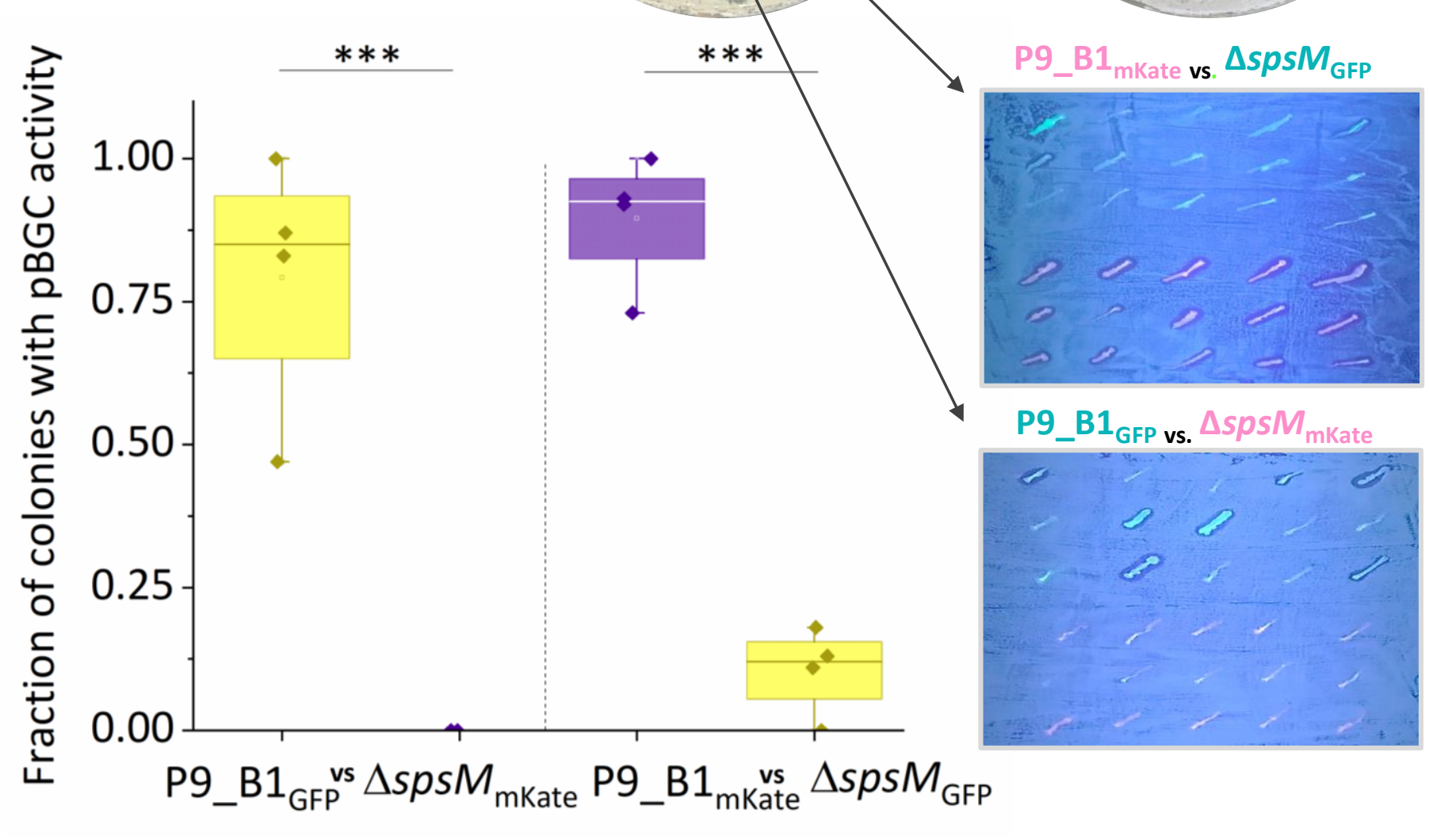




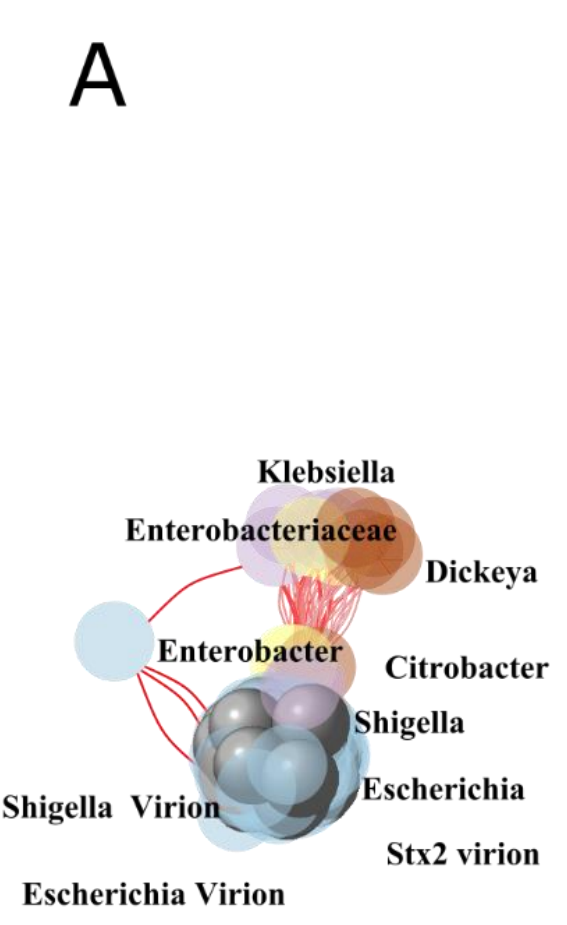

Lactobacillus Virion Lactobacillus

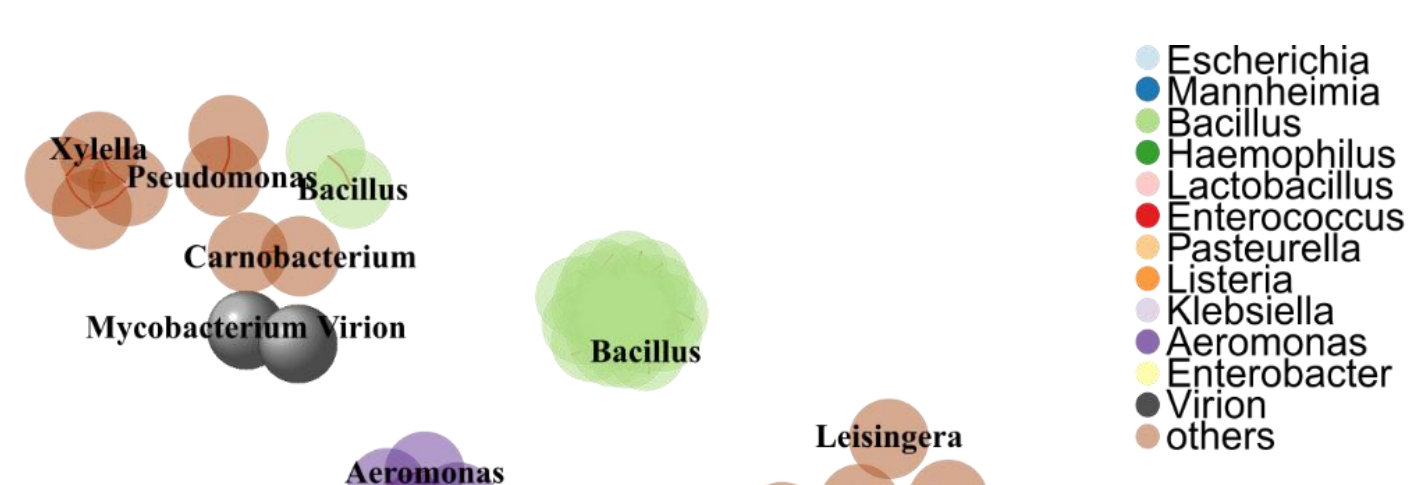

B

Nosalipuichastohacillus
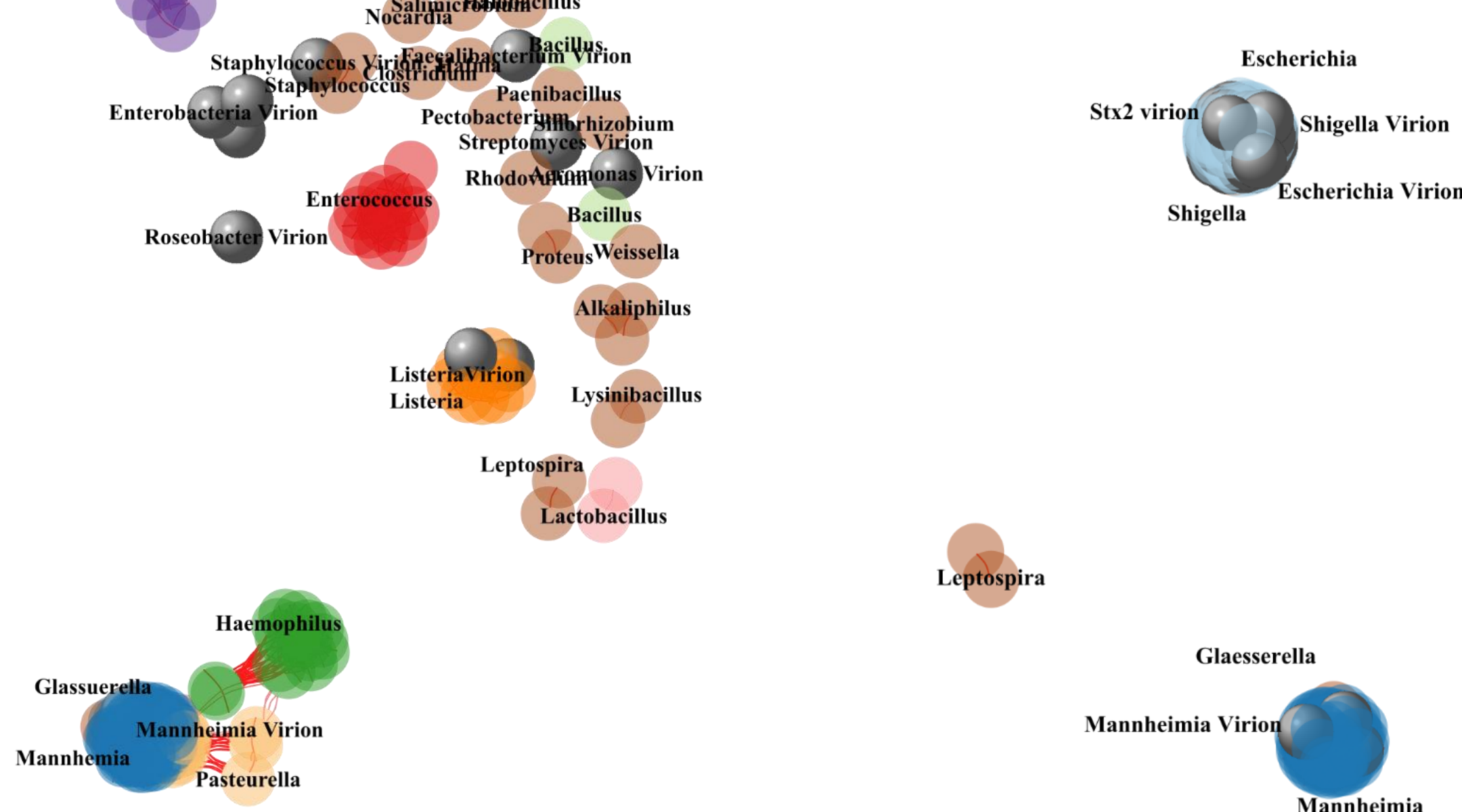

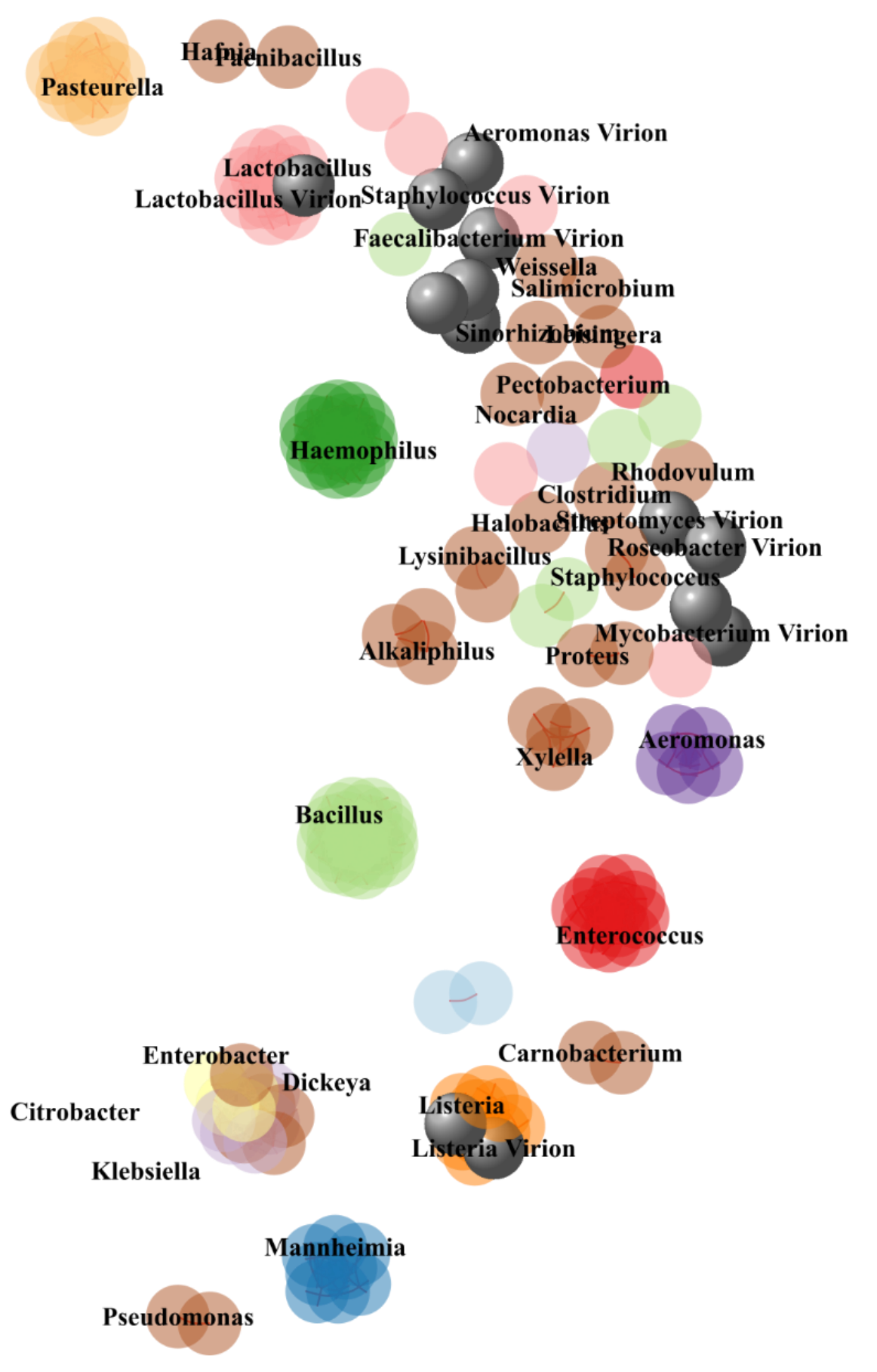

